\title{
Azithromycin-liposomes as a novel approach for localized therapy of cervicovaginal bacterial infections
}

This article was published in the following Dove Press journal: International Journal of Nanomedicine

\section{Željka Vanić' \\ Zora Rukavina' \\ Suvi Manner ${ }^{2}$ \\ Adyary Fallarero ${ }^{3}$ \\ Lidija Uzelac ${ }^{4}$ \\ Marijeta Kralj ${ }^{4}$ \\ Daniela Amidžić Klarićl \\ Anita Bogdanov ${ }^{5}$ \\ Tímea Raffai ${ }^{5}$ \\ Dezső Peter Virok ${ }^{5}$ \\ Jelena Filipović-Grčićl \\ Nataša Škalko-Basnet ${ }^{6}$ \\ 'Department of Pharmaceutical \\ Technology, Faculty of Pharmacy and \\ Biochemistry, University of Zagreb, \\ 10000 Zagreb, Croatia; ${ }^{2}$ Pharmaceutical \\ Sciences Laboratory, Faculty of Science \\ and Engineering, Åbo Akademi and \\ University of Helsinki, 20520 Turku, \\ Finland; ${ }^{3}$ Division of Pharmaceutical \\ Biosciences, Pharmaceutical Biology, \\ Faculty of Pharmacy, University of Helsinki, 000I4 Helsinki, Finland; \\ ${ }^{4}$ Department of Molecular Medicine, Ruđer Bošković Institute, 10000 Zagreb, \\ Croatia; ${ }^{5}$ Department of Medical \\ Microbiology and Immunobiology, \\ University of Szeged, 6720 Szeged, \\ Hungary; ${ }^{6}$ Drug Transport and Delivery \\ Research Group, Department of \\ Pharmacy, Faculty of Health Sciences, \\ University of Tromsø the Arctic \\ University of Norway, 5037 Tromsø, \\ Norway}

Correspondence: Željka Vanić

Department of Pharmaceutical

Technology, Faculty of Pharmacy and

Biochemistry, University of Zagreb, A.

Kovačića I, 10000 Zagreb, Croatia

Tel +385 | 6394762

Fax +385 I 46I 269 I

Email zvanic@pharma.hr
Background: Efficient localized cervicovaginal antibacterial therapy, enabling the delivery of antibiotic to the site of action at lower doses while escaping systemic drug effects and reducing the risk of developing microbial resistance, is attracting considerable attention. Liposomes have been shown to allow sustained drug release into vaginal mucosa and improve delivery of antibiotics to bacterial cells and biofilms. Azithromycin (AZI), a potent broad-spectrum macrolide antibiotic, has not yet been investigated for localized therapy of cervicovaginal infections, although it is administered orally for the treatment of sexually transmitted diseases. Encapsulation of AZI in liposomes could improve its solubility, antibacterial activity, and allow the prolonged drug release in the cervicovaginal tissue, while avoiding systemic side effects.

Purpose: The objective of this study was to develop AZI-liposomes and explore their potentials for treating cervicovaginal infections.

Methods: AZI-liposomes that differed in bilayer elasticity/rigidity and surface charge were prepared and evaluated under simulated cervicovaginal conditions to yield optimized liposomes, which were assessed for antibacterial activity against several planktonic and biofilmforming Escherichia coli strains and intracellular Chlamydia trachomatis, ex vivo AZI vaginal deposition/penetration, and in vitro cytotoxicity toward cervical cells.

Results: Negatively charged liposomes with rigid bilayers (CL-3), propylene glycol liposomes (PGL-2) and deformable propylene glycol liposomes (DPGL-2) were efficient against planktonic E. coli ATCC 700928 and K-12. CL-3 was superior for preventing the formation of $E$. coli ATCC 700928 and K-12 biofilms, with $\mathrm{IC}_{50}$ values (concentrations that inhibit biofilm viability by $50 \%$ ) up to 8 -fold lower than those of the control (free AZI). DPGL-2 was the most promising for eradication of already formed $E$. coli biofilms and for treating $C$. trachomatis infections. All AZI-liposomes were biocompatible with cervical cells and improved localization of the drug inside vaginal tissue compared with the control.

Conclusion: The performed studies confirm the potentials of AZI-liposomes for localized cervicovaginal therapy.

Keywords: vaginal drug delivery, biofilm, Escherichia coli, Chlamydia trachomatis, cervical cells, biocompatibility

\section{Introduction}

Bacterial cervicovaginal diseases, such as sexually transmitted Chlamydia trachomatis infections, recurrent bacterial vaginosis, and urogenital infections caused by Escherichia coli, represent a major public health problem with a substantial and expanding economic burden on the healthcare system. Vaginal E. coli strains are 
considered to be a reservoir for vaginal and/or endocervical colonization in pregnant women, which may result in progression of urinary tract, intra-amniotic and postpartum infections. Consequently, maternal or fetal complications can occur, including postpartum endometritis, bacteremia or sepsis. ${ }^{1}$ Untreated C. trachomatis infections may lead to infertility, adverse pregnancy outcomes, fetal loss and an increased risk of HIV transmission. ${ }^{2}$

The recommended treatment regimens for genital E. coli and C. trachomatis infections include oral administration of antibiotics. ${ }^{3,4}$ However, the incidence of antibiotic-related side effects and concerns about bacterial resistance make it necessary to consider other modes of tackling cervicovaginal infections, such as those based on localized cervicovaginal therapy.

Application of liposomally encapsulated antibiotics presents a promising strategy for enhanced drug delivery to bacterial cells and biofilms. ${ }^{5-7}$ Due to their phospholipid composition, liposomes are of particular interest regarding the safety issue and targeting ability. ${ }^{8}$ Tuning the physicochemical properties of liposomes, such as bilayer composition and fluidity, size, surface charge and coating, allows the development of drug-containing liposomes for localized therapy. ${ }^{9,10}$

Azithromycin (AZI) is a potent, broad-spectrum macrolide antibiotic that is available in dosage forms for oral, ophthalmic and parenteral administration. It is recommended for the treatment of respiratory, skin and soft tissue infections, including sexually transmitted bacterial diseases caused by $C$. trachomatis and Neisseria gonorrhoeae. ${ }^{11,12}$ AZI is usually available as a dihydrate (Mw 785) and is characterized by its limited solubility in water $(\log P=3.98) .{ }^{13}$ Incorporation of AZI into liposomes could increase its solubility, favor its cervicovaginal biocompatibility, and enable prolonged AZI release, permitting higher local drug concentrations.

Although AZI-liposomes have been tested against several pathogens, ${ }^{14-18}$ to the best of our knowledge, they have not been investigated for localized treatment of vaginal infections, and conventional topical formulations of AZI are not available for vaginal administration.

Therefore, for the first time, we investigated the potential of several types of AZI-liposomes, ie, conventional (CLs), propylene glycol (PGLs) and deformable propylene glycol liposomes (DPGLs), for the treatment of cervicovaginal infections. The different AZI-liposomes were characterized for the physical properties, in vitro release under simulated cervicovaginal conditions, and storage stability. The optimized liposomes were examined for ex vivo permeability on porcine vaginal mucosa, and their in vitro antibacterial activity was tested against several strains of biofilm-forming E. coli and intracellular C. trachomatis. Finally, the biocompatibility of the different AZI-liposomes was estimated in an in vitro cervical cell model.

To the best of our knowledge, AZI-liposomes have not yet been assessed against $C$. trachomatis and biofilmforming $E$. coli, and their in vitro compatibility with cervical cells has not yet been examined. Moreover, this study is the first to employ direct quantitative polymerase chain reaction (qPCR) measurements to study the antichlamydial effect of liposomally encapsulated antibiotics. Finally, the use of monoacyl phosphatidylcholine as an edge activator for the preparation of DPGLs represents a novel approach for the development of liposomes with elastic bilayers.

\section{Materials and methods Materials}

Egg phosphatidylcholine (EPC), egg phosphatidylglycerol sodium salt (EPG), hydrogenated soybean phosphatidylcholine (SPC-3) and monoacyl phosphatidylcholine from soybean (SLPC-80) were kindly donated by Lipoid GmbH (Ludwigshafen, Germany). AZI in the form of a dihydrate was a gift from PLIVA Croatia Ltd. (Zagreb, Croatia). Sephadex G-50, urea, glucose, lactic acid, Dulbecco's modified Eagle's medium, Minimum Essential Medium with Earle's salts, L-glutamine, fetal bovine serum and bovine serum albumin were obtained by Sigma-Aldrich (St. Louis, USA). Acetonitrile, ethanol and methanol were of HPLC grade and purchased from BDH Prolabo (Lutterworth, UK). Luria-Bertani (LB) agar and LB broth were obtained from Fisher Scientific (Leicestershire, UK) and Serva Electrophoresis GmbH (Heidelberg, Germany), respectively. Heat-inactivated fetal bovine serum was purchased from Euroclone (Pero, Italy). All other solvents or chemicals used in this research were of analytical grade and obtained from Kemika (Zagreb, Croatia) or SigmaAldrich.

A solution of $0.01 \mathrm{M}$ phosphate-buffered saline (PBS, $\mathrm{pH}$ 7.5) was prepared and filtered according to Rukavina et $\mathrm{al}^{17}$. Vaginal fluid simulant (VFS) was made by dissolving $0.222 \mathrm{~g}$ of $\mathrm{Ca}(\mathrm{OH})_{2}, 1.40 \mathrm{~g}$ of $\mathrm{KOH}, 3.51 \mathrm{~g}$ of $\mathrm{NaCl}, 2 \mathrm{~g}$ of lactic acid, $1 \mathrm{~g}$ of acetic acid, $0.16 \mathrm{~g}$ of glycerol, $0.4 \mathrm{~g}$ of urea, $5 \mathrm{~g}$ of glucose and $0.018 \mathrm{~g}$ of bovine serum albumin in demineralized water (up to $1000 \mathrm{ml}$ ) and adjusting the $\mathrm{pH}$ to 4.5 by the addition of $0.1 \mathrm{M} \mathrm{HCl}^{19}{ }^{19}$ Commercially available PBS, 
pH 7.3-7.5 (Lonza, Verviers, Belgium), was used in antibacterial assays against $E$. coli.

\section{Preparation of AZI-liposomes}

CLs were prepared by a modified proliposome method. ${ }^{20}$ In brief, phospholipids and the drug (Table 1) were dissolved in warm ethanol $(300 \mathrm{mg})$, followed by the addition

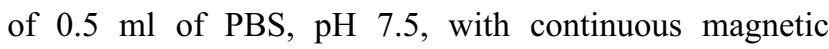
stirring $\left(600 \mathrm{rpm}, 60^{\circ} \mathrm{C}\right)$ to yield an initial proliposome mixture. This mixture was cooled to room temperature and then transformed into a liposomal dispersion by slow

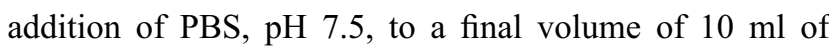
liposome dispersion. The dispersion was stirred $(600 \mathrm{rpm})$ for approximately $45 \mathrm{~min}$ at room temperature, while in the case of liposomes embedding SPC-3 (denoted as CL-2 and CL-3), the whole procedure was performed at approximately $50^{\circ} \mathrm{C}$.

For the preparation of elastic liposomes (PGLs and DPGLs), the selected phospholipids and AZI (Table 1) were dissolved in propylene glycol using a magnetic stirrer $\left(600 \mathrm{rpm}, 50^{\circ} \mathrm{C}\right.$ ). Then, $1 \mathrm{ml}$ of PBS, pH 7.5 (heated to the same temperature) was added. The mixture was stirred for approximately $2 \mathrm{~min}\left(50^{\circ} \mathrm{C}\right)$ and diluted to a liposomal

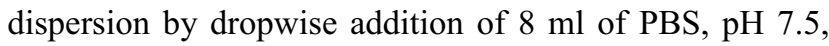
with continuous magnetic stirring (45 min).

The corresponding empty liposomes were prepared under the same conditions, omitting AZI, and were used as controls in in vitro antibacterial and biocompatibility studies.

All the liposomal dispersions were hand-extruded (LiposoFast Basic, Avestin, Canada) through $400 \mathrm{~nm}$ polycarbonate membranes ( 3 cycles) at room temperature and stored at $4^{\circ} \mathrm{C}$. The extrusion of CL-2 and CL-3 was performed at $50^{\circ} \mathrm{C}$.

\section{Size and zeta potential measurements}

The mean diameters, polydispersity indexes (PDIs) and zeta potentials of all the prepared liposomes were determined on a Zetasizer 3000HS (Malvern Instruments, Malvern, UK) at $25^{\circ} \mathrm{C}$ using a scattering angle of $90^{\circ}$ (size measurements) and a capillary cell with an optical modulator at $1,000 \mathrm{~Hz}$ (zeta potential measurements). Prior to the measurements, the instrument was calibrated with Malvern's size and zeta potential standards. The liposomal samples were prepared by diluting the liposomal dispersions with PBS, $\mathrm{pH} 7.5$, to achieve appropriate count rates. $^{21}$ The measurements were also performed in VFS, $\mathrm{pH} 4.5$, at $37^{\circ} \mathrm{C}$ and $25^{\circ} \mathrm{C}$.

\section{Determination of liposome bilayer elasticity/rigidity}

The elasticities/rigidities of the different AZI-liposome bilayers were determined using a previously reported method. ${ }^{22}$ In brief, liposomes were extruded through a $100 \mathrm{~nm}$ pore-sized membrane $\left(r_{p}\right)$ for $5 \mathrm{~min}$ at an external pressure of 2.5 bar. The mass of the extruded liposomes $(\mathrm{J})$ and the mean diameter of the liposomes after extrusion $\left(\mathrm{r}_{\mathrm{v}}\right)$ were determined. The degree of bilayer elasticity (E) was calculated by applying the following equation: ${ }^{21}$

$$
E=J \cdot\left(r_{v} / r_{p}\right)^{2}
$$

\section{Encapsulation efficiency determination and quantification of $A Z I$}

The encapsulation of AZI in the different liposomes was determined after separation of unencapsulated AZI by a minicoloumn centrifugation method. ${ }^{23}$ In brief, minicolumns filled with Sephadex G-50 gel were dried by

Table I Composition of liposomes

\begin{tabular}{|l|l|l|l|l|l|l|l|l|}
\hline Liposomes type & Code & EPC (mg) & EPG (mg) & SPC-3 (mg) & SLPC-80 (mg) & AZI (mg) & PG (g) & PBS, pH 7.5 (mI) \\
\hline CLs & CL-I & 190 & 10 & - & - & 30 & - & 10 \\
& CL-2 & 150 & - & 50 & - & 30 & - & 10 \\
& CL-3 & 140 & 10 & 50 & - & 30 & - & 10 \\
\hline PGLs* & PGL-I & 200 & - & - & - & 30 & 1 & 9 \\
& PGL-2 & 190 & 10 & - & - & 30 & 1 & 9 \\
\hline \multirow{2}{*}{ DPGL** } & DPGL-I & 170 & - & - & 30 & 30 & 1 & 9 \\
& DPGL-2 & 160 & 10 & - & 30 & 30 & 1 & 9 \\
\hline
\end{tabular}

Notes: The final volume of liposome dispersion was $10 \mathrm{ml}$. *Elastic liposomes.

Abbreviations: AZI, azithromycin; CLs, conventional liposomes; DPGLs, deformable propylene glycol liposomes; EPC, egg phosphytidylcholine; EPG, egg phosphatidylglycerol sodium salt; PBS, phosphate-buffered saline; PG, propylene glycol; PGLs, propylene glycol liposomes; SLPC-80, soybean monoacyl phosphatidylcholine; SPC-3, hydrogenated soybean phosphatidylcholine. 
centrifugation $(2,000 \mathrm{rpm}, 3 \mathrm{~min})$. Then, $0.4 \mathrm{ml}$ of liposome dispersions were cautiously applied to the top of the gels, followed by centrifugation under the same conditions to obtain AZI-liposomes in the centrifuge tubes, which were saved for assessment. Subsequently, $0.5 \mathrm{ml}$ of PBS, $\mathrm{pH} 7.5$, was added to each minicolumn, which was again centrifuged, and the eluates were collected. The last procedure, including rinsing the minicolumns with PBS, was repeated until all unencapsulated AZI was removed.

Quantification of AZI, both unencapsulated and liposomally encapsulated, was performed by HPLC under the previously described conditions. ${ }^{17}$

The encapsulation of AZI was expressed as the encapsulation efficiency (\%), ie, the percentage of encapsulated AZI compared to the original drug concentration and the amount of encapsulated drug per mass of phospholipids used.

\section{In vitro release studies}

The release of AZI from the different liposomes, which were previously separated from the unencapsulated drug, was assessed under conditions simulating vaginal environment using the so-called bag dialysis method. AZI-liposomes (corresponding to $1 \mathrm{mg}$ of $\mathrm{AZI}$ ) were placed in a dialysis bag ( $\mathrm{Mw}$ cut off 12-14,000 Da, Medicell Membranes Ltd., London, UK) and dialyzed against $25 \mathrm{ml}$ of VFS (pH 4.5) with constant stirring $(50 \mathrm{rpm})$ and incubation at $37^{\circ} \mathrm{C}$. Aliquots $(2 \mathrm{ml})$, containing the released drug, were collected at predetermined time intervals (1, 2, 3, 4, 5, 6 and $24 \mathrm{~h})$ and immediately replaced with the same volume of fresh VFS, $\mathrm{pH} 4.5$. The amount of released drug was determined by HPLC. ${ }^{17}$

To investigate the influence of $\mathrm{pH}$ on AZI release and to mimic cervical conditions, studies were also performed in PBS, pH 7.5. Free AZI dissolved in a mixture of ethanol and demineralized water $(6 / 4, v / v)$ served as a control and was examined at $\mathrm{pH} 7.5$.

\section{Storage stability studies}

The changes in the physical properties of AZI-liposomes (mean diameters, PDIs and zeta potentials) were monitored during storage of the liposome dispersions for 8 months at $4^{\circ} \mathrm{C}$. The measurements were performed at $25^{\circ} \mathrm{C}$.

\section{Ex vivo vaginal deposition/penetration studies}

Porcine vaginal mucosa was used to evaluate the deposition/ penetration of AZI from the selected AZI-liposomes. The vaginal tissues from the adult animals (approximately 1 year old) were received as waste from a local slaughterhouse. The vaginal tissue was carefully separated from the underlying tissue, cleaned with physiological solution $(0.9 \% \mathrm{NaCl})$, cut into smaller pieces (approximately $3 \times 3 \mathrm{~cm}$ ) and frozen at $-20^{\circ} \mathrm{C}$. Approximately $30 \mathrm{~min}$ prior to the experiments, the vaginal tissue was defrosted and pre-equilibrated in VFS for $15 \mathrm{~min}$. The vaginal tissue (900-1,050 $\mu \mathrm{m}$ thick) was fixed between the donor and acceptor chamber of the Franz cell $\left(3.14 \mathrm{~cm}^{2}\right.$, surface area), with the vaginal epithelium facing the donor chamber. The acceptor chamber $(15 \mathrm{ml})$ was filled with PBS, $\mathrm{pH} 7.5$, and constantly stirred (200 rpm). The whole system was maintained at $37 \pm 1^{\circ} \mathrm{C}$. Samples of AZIliposomes or the control (free AZI, 6/4, ethanol/water solution), equivalent to $1 \mathrm{mg}$ of $\mathrm{AZI}$, were added to the donor chamber and covered with parafilm. The acceptor medium $(500 \mu \mathrm{l})$ was sampled at intervals of 1, 2, 3, 4, 5, 6 and $24 \mathrm{~h}$ and immediately replenished with the same volume of fresh PBS. After $24 \mathrm{~h}$, the liquid (if remained) in the donor chamber was removed by pipetting, and the vaginal surface was carefully washed with methanol. Both fractions were mixed together and dissolved in methanol, indicating nonpenetrating AZI. The vaginal tissue was then shred and extracted with methanol ( $4 \mathrm{~h}$, agitation) to determine the drug that remained inside the vaginal tissue. The AZI content in all the collected samples was quantified by HPLC. ${ }^{17}$

\section{Antibacterial assays against E. coli Bacterial strains and growth conditions}

The three E. coli strains (ATCC 8739, ATCC 700928 and $\mathrm{K}-12$ ) were stored in $20 \%$ glycerol stocks at $-70^{\circ} \mathrm{C}$. Working stocks of each strain were prepared on LB agar plates and stored at $+4^{\circ} \mathrm{C}$. For liquid cultures, a $1 \mu 1$ inoculation loop full of colonies was transferred to $3 \mathrm{ml}$ of LB broth and incubated under aerobic conditions $\left(37^{\circ} \mathrm{C}\right.$, $220 \mathrm{rpm}$ ) overnight. The overnight cultures were diluted in LB broth and incubated until they reached exponential growth, corresponding to approximately $10^{8}$ colony forming units per milliliter $(\mathrm{CFU} / \mathrm{ml})$. The bacterial concentration was estimated by measuring the turbidity at $595 \mathrm{~nm}$ using a Varioskan Flash Multimode plate reader (Thermo Fisher Scientific, Vantaa, Finland) and further confirmed by serially diluting and plating the cultures on LB agar for the determination of $\mathrm{CFU} / \mathrm{ml}$.

\section{In vitro antibacterial activity against biofilm and planktonic E. coli}

The anti-biofilm activity of the selected AZI-liposomes (CL-3, PGL-2 and DPGL-2) toward E. coli strains was 
tested and compared to the activity of free AZI (6/4, v/v, ethanol/water solution) and the corresponding empty liposomes (without encapsulated AZI).

Two-fold dilution series of AZI-liposomes and free AZI (solution) were prepared in LB and tested at eight concentrations ranging from $0.61 \mu \mathrm{g} / \mathrm{ml}$ to $78.50 \mu \mathrm{g} / \mathrm{ml}$. The studies were performed in two modes, pre- and postbiofilm formation. ${ }^{24}$ In both cases, exponentially grown bacterial cultures, diluted to a concentration of $10^{6} \mathrm{CFU} /$ $\mathrm{ml}$, were used. Briefly, in the pre-exposure mode of the assay, bacteria and AZI samples were simultaneously added to Nunc $^{\text {TM }}$ 96-well polystyrene microplates (Nunclon $^{\mathrm{TM}}$ Delta surface) at a final volume of $200 \mu \mathrm{l}$. The plates were incubated at $37^{\circ} \mathrm{C}, 200 \mathrm{rpm}$, for $18 \mathrm{~h}$. For the postexposure mode, bacteria were initially added to the microtiter well plates $(200 \mu \mathrm{l})$ and allowed to form biofilms for $18 \mathrm{~h}\left(37^{\circ} \mathrm{C}, 200 \mathrm{rpm}\right)$. After $18 \mathrm{~h}$, the planktonic bacterial phase was discarded, and the AZI samples (prepared as described above) and fresh culture medium were added to the wells. The plates were further incubated for $24 \mathrm{~h}\left(37^{\circ} \mathrm{C}, 200 \mathrm{rpm}\right)$. The samples were tested in duplicate in four independent experiments. Empty liposomes (without AZI) were always included as a negative control, and a mixture of ethanol and water was included as a solvent control, in addition to the wells that contained only medium and untreated bacteria.

Anti-biofilm activity was quantified using a resazurin (redox) staining assay. ${ }^{24}$ At the end of the incubation periods with the compounds (in the pre-exposure and postexposure modes), the planktonic phase was removed, and the biofilms were washed once with PBS. Resazurin solution in PBS $(20 \mu \mathrm{M})$ was added to the wells $(200 \mu \mathrm{l})$, and the plates were incubated at room temperature for $1 \mathrm{~h}$. Fluorescence was measured $(\lambda \mathrm{ex}=560 \mathrm{~nm}, \lambda \mathrm{em}=590 \mathrm{~nm})$ using a Varioskan Flash Multimode plate reader using a top fluorescence reading. Anti-biofilm activity was determined as the inhibition percentage compared to untreated biofilms. The concentrations of the samples that inhibited biofilm viability by $50 \%\left(\mathrm{IC}_{50}\right)$ were calculated from eight concentration points via nonlinear regression analysis using GraphPad Prism 6.0 software.

Additionally, antibacterial activity against planktonic bacteria was assessed spectrophotometrically. At the end of the $18 \mathrm{hr}$ incubation period (in the pre-exposure mode of the assay), bacterial turbidity was recorded at $620 \mathrm{~nm}$ using a Varioskan Flash Multimode plate reader. Antibacterial effects were determined as inhibition percentages compared to untreated bacteria. The concentrations of the samples that inhibited bacterial growth by $50 \%$ (minimum inhibitory concentrations, $\mathrm{MIC}_{50}$ ) were determined as described above.

\section{In vitro antibacterial assay against intracellular C. trachomatis Bacterial strain}

The C. trachomatis serovar D (UW-3/CX, ATCC) strain was propagated and partially purified according to previously described methods. ${ }^{25,26}$ Briefly, DEAE-dextran (45 $\mathrm{mg} / \mathrm{ml}$ in Hanks' balanced salt solution) treated McCoy cells were infected with $C$. trachomatis serovar $\mathrm{D}$, and incubated for $48 \mathrm{~h}$ in Minimal Essential Medium with Earle salts supplemented with $10 \%$ fetal bovine serum, $8 \mathrm{mmol} / 1 \mathrm{HEPES}, 0.5 \%$ glucose, $1 \times$ nonessential amino acids, $2 \mathrm{mmol} / \mathrm{l}$ L-glutamine, $25 \mu \mathrm{g} / \mathrm{ml}$ gentamicin and $1 \mu \mathrm{g} / \mathrm{ml}$ cycloheximide. Infected cells were lysed by 2 freeze-thaw cycles in sucrose-phosphate-glutamic acid buffer $48 \mathrm{~h}$ post-infection. The cell lysate was centrifuged (800 g, $10 \mathrm{~min}$ ) and the supernatant containing the chlamydial elementary bodies were used for subsequent experiments. The titer of the chlamydial stock was calculated by infecting McCoy cells and counting the inclusion numbers $48 \mathrm{~h}$ post-infection with a monoclonal anti-chlamydia LPS antibody (AbD Serotec, Oxford, United Kingdom) and a FITC-labeled anti-mouse IgG (SigmaAldrich, St. Louis, MO).

Culture and infection of HeLa cells with $C$. trachomatis in the presence of AZI-liposomes and free AZI

HeLa 229 cells (ATCC) (40,000 cells/well) were cultured in 96-well microtiter plates (Sarstedt, Nümbrecht, Germany) in $100 \mu \mathrm{l}$ of Minimum Essential Medium supplemented with Earle's salts, 10\% heat-inactivated fetal bovine serum, $1 \times$ nonessential amino acids, $8 \mathrm{mmol} / \mathrm{l}$ HEPES, $2 \mathrm{mmol} / \mathrm{l}$ L-glutamine, $1 \mu \mathrm{g} / \mathrm{ml}$ fungisone and $25 \mu \mathrm{g} / \mathrm{ml}$ gentamycin. The cells were incubated overnight at $37^{\circ} \mathrm{C}$ in $5 \% \mathrm{CO}_{2}$ to obtain a $90 \%$ confluent cell layer. ${ }^{27}$ HeLa cells were washed twice with $100 \mu \mathrm{l}$ of PBS, pH 7, per well and infected with $C$. trachomatis (multiplicity of infection, MOI 0.2) as described previously. ${ }^{28}$ After infection, the HeLa cells were washed twice with PBS and cultured in cycloheximide-free medium supplemented with a dilution series of the applied AZI samples $(n=3)$. A concentration range of $0.5-0.0002 \mu \mathrm{g} / \mathrm{ml} \mathrm{AZI} \mathrm{for} \mathrm{AZI-}$ liposomes (CL-3, PGL-2 and DPGL-2) and free AZI (solution) was tested. The cells were incubated for $48 \mathrm{~h}$ at $37^{\circ} \mathrm{C}$ in $5 \% \mathrm{CO}_{2}$. 
Cell lysis and direct quantitative polymerase chain reaction ( $q P C R)$ measurements

Direct qPCR was performed according to Eszik et $\mathrm{al}^{28}$. Briefly, $48 \mathrm{~h}$ post infection, the HeLa cells were washed twice with $100 \mu \mathrm{l} /$ well PBS. Finally, $100 \mu \mathrm{l}$ of Milli-Q water (Millipore, Billerica, MA, USA) was added to the wells, and two freeze-thaw cycles were performed. The mixed cell lysates were used as templates in qPCR for chlamydial genomic DNA quantitation. qPCR was performed using the C. trachomatis pykF gene-specific primers in a BioRad CFX96 real-time system (Bio-Rad, Hercules, CA, USA). The primer sequences were: pykF forward: 5'GTTGCCAACGCCATTTACGATGGA-3', pykF reverse: 5'-TGCATGTACAGGATGGGCTCCTAA-3'. Two microliters of 5x HOT FIREPol ${ }^{\circledR}$ EvaGreen ${ }^{\circledR}$ qPCR Supermix (Solis BioDyne, Tartu, Estonia), 10 pmol of qPCR primers, $1 \mu \mathrm{l}$ of cell lysate template and $5 \mu \mathrm{l}$ of Milli-Q water were used per reaction. The qPCR cycle parameters were as follows: $12 \mathrm{~min}$ at $95^{\circ} \mathrm{C}$ for polymerase activation, 40 cycles at $95^{\circ} \mathrm{C}$ for $20 \mathrm{~s}$ and $64^{\circ} \mathrm{C}$ for $1 \mathrm{~min}$, and a melting curve analysis step for specificity testing. Cycle threshold (Ct) levels were calculated for each sample. Minimum inhibitory concentration (MIC) values for C. trachomatis were identified as previously described. ${ }^{28}$

\section{In vitro biocompatibility studies \\ Cell culturing}

HeLa cells were cultured as monolayers and maintained in Dulbecco's modified Eagle's medium supplemented with $10 \%$ fetal bovine serum, $2 \mathrm{mM}$ L-glutamine, $100 \mathrm{U} / \mathrm{ml}$ penicillin and $100 \mu \mathrm{g} / \mathrm{ml}$ streptomycin in a humidified atmosphere with $5 \% \mathrm{CO}_{2}$ at $37^{\circ} \mathrm{C}$.

\section{Cell toxicity study}

The cells were inoculated onto 96-well microtiter plates at concentrations of $1.6 \times 10^{4}$ cells $/ \mathrm{ml}$. AZI-liposomes (CL-3, PGL-2 and DPGL-2) or free AZI (6/4, v/v, ethanol/water solution) was added the next day at five dilutions (39.25 $\mu \mathrm{g} / \mathrm{ml}, 19.63 \mu \mathrm{g} / \mathrm{ml}, 9.81 \mu \mathrm{g} / \mathrm{ml}$ and $2.45 \mu \mathrm{g} / \mathrm{ml}$ and $1.23 \mu \mathrm{g} / \mathrm{ml}$ ) and incubated with the cells for an additional $24 \mathrm{~h}\left(37^{\circ} \mathrm{C}, 5 \% \mathrm{CO}_{2}\right)$. The cell viability was evaluated by the MTT assay, a colorimetric assay system that measures the reduction of a tetrazolium component (MTT) into an insoluble formazan product by the mitochondria of viable cells. ${ }^{29}$ The absorbance was determined spectrophotometrically at $570 \mathrm{~nm}$ on a microplate reader (Multiscan, Thermo Labsystems, Waltham, MA, USA) and was directly proportional to the cell viability. The cell viability of the treated cells was expressed as a percentage compared to the untreated control cells. Each result represents an average value from at least three separate experiments performed in quadruplicate.

\section{Statistical analysis}

Statistical data analyses were performed using the GraphPad 5.0 Prism program (GraphPad Software, Inc., San Diego, USA). Student's $t$-test was applied for comparisons between two groups, and means were considered significantly different when $p<0.05$. One-way ANOVA and Tukey's multiple comparison test, with $p<0.05$ set as the minimal level of significance, were applied for comparisons of three or more groups.

\section{Results and discussion Characterization of liposomes}

A suitable characterization of liposomes is crucial for obtaining reliable data with high translational output. ${ }^{30}$ Namely, the physicochemical properties of liposomes influence their stability in biological environments and during storage, determine the pharmacokinetic properties of the encapsulated drug and contribute toward their behavior within the biological milieu, subsequently affecting the efficiency of therapy. Therefore, the first phase in the present study focused on assessing the physical characteristics of AZI-liposomes that differed in terms of bilayer composition, the presence of cosolvent and the edge activator (Table 1). Liposomes were evaluated for size and surface charge (Tables 2 and 3), bilayer elasticity (Table 4) and encapsulation efficiency (Figure 1).

As presented in Table 2, AZI-liposomes composed of only neutral phospholipids (CL-2, PGL-1 and DPGL-1) were significantly larger $(544-840 \mathrm{~nm})$, with broad size distributions (PDI $>0.49$ ), than the corresponding liposomes containing negatively charged EPG (CL-3, PGL-2 and DPGL-2) $(t$-test, $p<0.05)$. Extrusion of all AZI-liposomes significantly decreased their mean diameters (189$261 \mathrm{~nm})$ and PDIs $(0.17-0.27)(t$-test, $p<0.05)$. The only exception was PGL-1 with PDI $>0.7$, indicating a tendency of PGL-1 to form aggregates in the presence of propylene glycol and the absence of a negatively charged phospholipid, which both affect the size of the liposomes. Comparison of the various types of negatively charged liposomes (CL-1, CL-3, PGL-2 and DPGL-2) revealed DPGL-2 as the smallest. These results are in agreement with previously published findings showing the ability of 
Table 2 Size and surface characteristics of the AZI-liposomes

\begin{tabular}{|c|c|c|c|c|c|}
\hline \multirow[t]{2}{*}{ Liposomes } & \multicolumn{2}{|l|}{ Originally prepared } & \multicolumn{2}{|l|}{ Extruded $(3 \times 400 \mathrm{~nm})$} & \multirow[t]{2}{*}{ Zeta potential $(\mathrm{mV})$} \\
\hline & Mean diameter $(\mathrm{nm})$ & PDI & Mean diameter $(\mathrm{nm})$ & PDI & \\
\hline CL-I & $351 \pm 3$ & $0.27 \pm 0.02$ & $259 \pm I^{*}$ & $0.17 \pm 0.01 *$ & $-66.4 \pm 1.3$ \\
\hline CL-2 & $840 \pm 11$ & $0.63 \pm 0.08$ & $221 \pm 2 *$ & $0.26 \pm 0.10^{*}$ & $-21.6 \pm 0.6$ \\
\hline CL-3 & $466 \pm 3$ & $0.24 \pm 0.03$ & $262 \pm 3 *$ & $0.18 \pm 0.03$ & $-61.5 \pm 0.7$ \\
\hline PGL-I & $545 \pm 14$ & $0.56 \pm 0.01$ & $34 I \pm 27^{\# *}$ & $0.74 \pm 0.08^{*}$ & $-18.2 \pm 0.5$ \\
\hline PGL-2 & $529 \pm 10$ & $0.24 \pm 0.01$ & $222 \pm 2 *$ & $0.20 \pm 0.02 *$ & $-55.8 \pm 0.9$ \\
\hline DPGL-I & $574 \pm 8$ & $0.49 \pm 0.09$ & $219 \pm 1^{*}$ & $0.27 \pm 0.0 I^{*}$ & $-17.8 \pm 0.6$ \\
\hline DPGL-2 & $287 \pm 3$ & $0.3 I \pm 0.01$ & $189 \pm I^{*}$ & $0.22 \pm 0.02 *$ & $-53.1 \pm 0.5$ \\
\hline
\end{tabular}

Notes: The values are the mean \pm S.D.; $n=3$ (mean diameter, PDI); $n=5$ (zeta potential); ${ }^{*}$ Estimated size due to high PDI value $(>0.7)$. $*$ Statistically significant compared to the originally prepared liposomes $(t$-test, $p<0.05)$.

Abbreviation: PDI, polydispersity index.

Table 3 Characteristics of the AZI-liposomes at simulated vaginal conditions

\begin{tabular}{|l|l|l|l|}
\hline Liposomes & Mean diameter (nm) & PDI & Zeta potential (mV) \\
\hline CL-I & $276 \pm 5^{\mathrm{a}}$ & $0.19 \pm 0.02^{\mathrm{a}}$ & $-0.6 \pm 0.3^{\mathrm{a}}$ \\
& $270 \pm 3^{\mathrm{b}}$ & $0.20 \pm 0.02^{\mathrm{b}}$ & $-0.4 \pm 0.1^{\mathrm{b}}$ \\
\hline CL-2 & $392 \pm 24^{\mathrm{a}}$ & $0.46 \pm 0.08^{\mathrm{a}}$ & $4.1 \pm 0.4^{\mathrm{a}}$ \\
& $397 \pm 12^{\mathrm{b}}$ & $0.42 \pm 0.10^{\mathrm{b}}$ & $4.4 \pm 0.3^{\mathrm{b}}$ \\
\hline CL-3 & $323 \pm 4^{\mathrm{a}}$ & $0.22 \pm 0.02^{\mathrm{a}}$ & $-4.4 \pm 0.3^{\mathrm{a}}$ \\
& $331 \pm 3^{\mathrm{b}}$ & $0.21 \pm 0.09^{\mathrm{b}}$ & $-4.2 \pm 0.3^{\mathrm{b}}$ \\
\hline PGL-I & $382 \pm 7^{\mathrm{a}}$ & $0.77 \pm 0.09^{\mathrm{a}}$ & $4.4 \pm 0.3^{\mathrm{a}}$ \\
\hline PGL-2 & $389 \pm 9^{\mathrm{b}}$ & $0.73 \pm 0.08^{\mathrm{b}}$ & $5.1 \pm 0.4^{\mathrm{b}}$ \\
\hline DPGL-I & $274 \pm 4^{\mathrm{a}}$ & $0.15 \pm 0.03^{\mathrm{a}}$ & $-1.8 \pm 0.3^{\mathrm{a}}$ \\
& $276 \pm 3^{\mathrm{b}}$ & $0.17 \pm 0.08^{\mathrm{b}}$ & $-2.1 \pm 0.1^{\mathrm{b}}$ \\
\hline DPGL-2 & $262 \pm 5^{\mathrm{a}}$ & $0.21 \pm 0.10^{\mathrm{a}}$ & $3.8 \pm 0.3^{\mathrm{a}}$ \\
& $258 \pm 3^{\mathrm{b}}$ & $0.22 \pm 0.05^{\mathrm{b}}$ & $4.6 \pm 0.2^{\mathrm{b}}$ \\
\hline
\end{tabular}

Notes: ${ }^{\mathrm{a}} 37^{\circ} \mathrm{C} ;{ }^{\mathrm{b}} 25^{\circ} \mathrm{C}$. The extruded AZI-liposomes were dispersed in VFS, $\mathrm{pH} 4.5$, and the mean diameters, PDIs and zeta potentials were determined at $37^{\circ} \mathrm{C}$. The measurements were also performed at $25^{\circ} \mathrm{C}$ to investigate the effect of temperature on the physical properties of liposomes. The each value represents the mean \pm S.D. $(n=3$, mean diameter, PDI; $n=5$, zeta potential). Significant differences between the determined parameters were not obtained when experiments were carried out at 25 or $37^{\circ} \mathrm{C}(t$-test, $p>0.05)$.

Abbreviation: PDI, polydispersity index.

an edge activator to reduce the size of deformable liposomes, ${ }^{21,23}$ which might be attributed to the enhanced elasticity (flexibility) and decreased surface tension of the liposomes in the presence of single chain surfactants. ${ }^{31}$ Moreover, a decrease in the size of microemulsions containing an increased ratio of monoacyl phosphatidylcholine (used as the edge activator in DPGLs in the present study) has been confirmed by Hoppel et $\mathrm{al}^{32}$.

The zeta potential values (Table 2 ) followed the properties of the materials used for the preparation of liposomes. Liposomes prepared without EPG had zeta potentials between -17 and $-21 \mathrm{mV}$, while those containing EPG had strongly negative zeta potentials higher than $-50 \mathrm{mV}$, indicating the formation of physically stable liposome dispersions.

To resemble more closely the conditions AZI-liposomes will be exposed to, the evaluation of their physicochemical properties was also performed at simulated vaginal conditions (VFS, $\mathrm{pH} 4.5,37^{\circ} \mathrm{C}$ ). Moreover, to determine the possible effect of the temperature on the size and surface charge of AZI-liposomes, the experiments were also conducted at $25^{\circ} \mathrm{C}$.

The results presented in Table 3 demonstrate significant increase ( $t$-test, $p<0.05)$ in mean diameters of all AZI-liposomes because of their interaction with vaginal fluid 
Table 4 Liposome bilayer elasticity

\begin{tabular}{|l|l|l|l|}
\hline Liposomes & J (g) & $\mathbf{r}_{\mathbf{v}}(\mathbf{n m})$ & $\mathbf{E}$ \\
\hline CL-I & $1.61 \pm 0.2$ & $165 \pm 2$ & $4.4 \pm 0.3^{*}$ \\
CL-2 & $0.17 \pm 0.0$ & $185 \pm 3$ & $0.58 \pm 0.1$ \\
CL-3 & $0.19 \pm 0.1$ & $165 \pm 2$ & $0.52 \pm 0.2$ \\
PGL-I & $4.26 \pm 0.3$ & $178 \pm 1$ & $13.53 \pm 0.3^{*}$ \\
PGL-2 & $4.41 \pm 0.2$ & $169 \pm 2$ & $12.64 \pm 0.2^{*}$ \\
DPGL-I & $6.63 \pm 0.4$ & $154 \pm 2$ & $15.70 \pm 0.4^{*}$ \\
DPGL-2 & $7.91 \pm 0.3$ & $147 \pm 1$ & $17.02 \pm 0.3^{*}$ \\
\hline
\end{tabular}

Notes: The values are the mean \pm S.D. $(n=3)$. One-way ANOVA and Tukey's multiple comparison test, with $p<0.05$ set as the minimal level of significance, were applied for comparison of the $E$ values. *Statistically significant compared to CL-2 and CL-3 $(p<0.05)$.

Abbreviations: $\mathrm{E}$, degree of liposome bilayers elasticity; J, amount of extruded liposomes; $r_{v}$, average diameter of liposomes following extrusion under constant pressure.

components, in comparison to the corresponding values shown in Table 2. However, polydispersity indexes commonly remained unchanged despite of the used media (buffer, $\mathrm{pH}$ 7.5 or VFS, $\mathrm{pH} 4.5$ ), except for the CL-2 and PGL-1.

Similarly as the mean diameters, the zeta potentials of AZI-liposomes (Table 2) have significantly changed ( $t$-test, $p<0.05$ ) at mimicked vaginal conditions (Table 3 ). The originally, highly negative zeta potentials of CL-1, CL-3, PGL-2 and DPGL-2 decreased from approximately $-50 \mathrm{mV}$ (buffer, $\mathrm{pH}$ 7.5) to $-4 \mathrm{mV}$ (VFS, $\mathrm{pH} 4.5$ ), while AZI-liposomes with slightly negative surface charge (between -18 and $-21 \mathrm{mV}$ ) changed to slightly positive values $(4 \mathrm{mV})$. Interestingly, the temperature did not affect the size and surface properties of the liposomes ( $t$-test, $p>0.05)$.

Since the cervical tissue is characterized by neutral $\mathrm{pH}^{33}$ and considering the fact that the temperature had no impact on the physical properties of liposomes (Table 3), the physical properties of AZI-liposomes shown in Table 2 (obtained in the buffer, $\mathrm{pH} 7.5$ ) could be translated to mimicked cervical conditions.

The thermodynamic state of the liposome bilayers is responsible for their release pattern and permeation ability, as well as the interactions of liposomes with bacteria and biofilms. ${ }^{17,23,34}$ Liposome bilayer elasticity/rigidity was evaluated by applying an easy and reproducible method based on the passage of liposomes through 100-nm membranes under a constant external pressure (2.5 bar) for $5 \mathrm{~min}^{22}$ The results presented in Table 4 confirmed that PGLs and DPGLs are elastic liposomes, while CLs, particularly CL-2 and CL-3, exhibited pronounced rigid bilayers. In addition, the degree of bilayer elasticity (E) was confirmed to be commonly dependent on the quantity of extruded liposomes $(\mathrm{J})$ rather than their size following extrusion $\left(r_{v}\right)$ through $100-n m$ membranes $\left(r_{p}\right)$. Comparison of the calculated $\mathrm{E}$ values among the different CLs showed the lowest elasticity for CL-2 and CL-3 due to the presence of $25 \%(\mathrm{w} / \mathrm{w})$ hydrogenated soybean phosphatidylcholine (SPC-3) in the bilayers. In contrast, PGLs and DPGLs were characterized by flexible bilayers with $\mathrm{E}$ values between 12 and 17, whereas the elasticity of PGLs was somewhat less pronounced. The highest E value was confirmed for DPGL-2 (ANOVA, $p<0.05$ ), with a value almost 34 -fold higher than the E values of CL-2 and CL-3. The increased elasticity of DPGLs compared with PGLs is ascribed to the presence of $15 \%(\mathrm{w} / \mathrm{w})$ monoacyl phosphatidylcholine in DPGLs. These results are in accordance with previously published findings for DPGLs containing the same ratio of propylene glycol but with sodium deoxycholate as an edge activator. ${ }^{22}$

Properly balancing physical characteristics enables the development of liposomes with suitable encapsulation of AZI as a prerequisite for adequate therapeutic outcomes.

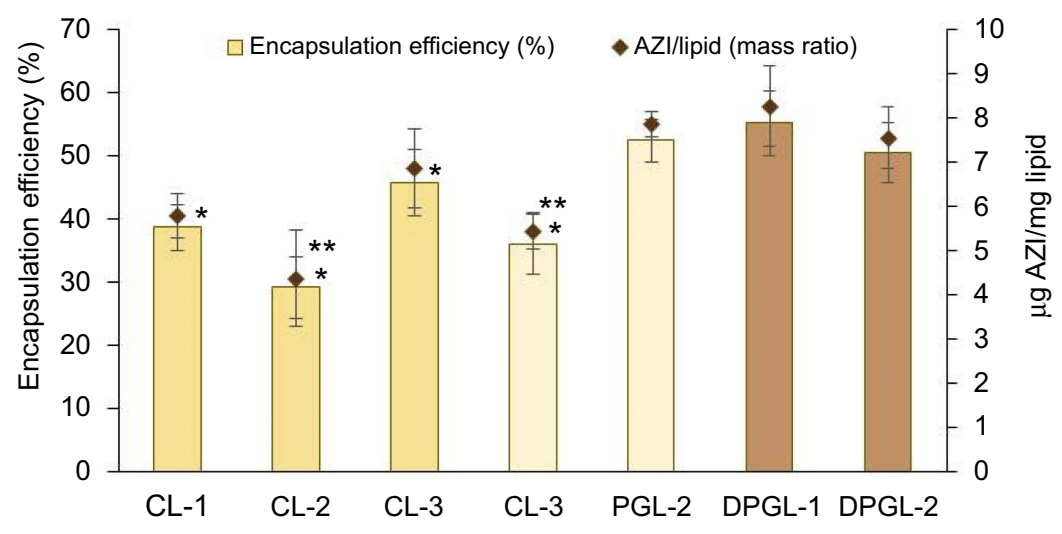

Figure I Encapsulation of AZI in different types of liposomes expressed as the encapsulation efficiency (\%) and the drug-per-lipid ratio.

Notes: The values indicate the mean \pm S.D. *Significantly different from DPGL-I (ANOVA, $p<0.05$ ). **Significantly different from the corresponding highly negatively surface-charged liposomes ( $t$-test, $p<0.05)$.

Abbreviation: AZI, azithromycin. 
Since our aim was topical vaginal therapy, liposomes with diameters of 200-300 nm are expected to be optimal. ${ }^{35}$

As shown in Figure 1, encapsulation of AZI differed between 29\% (4.4 $\mu \mathrm{g} \mathrm{AZI} / \mathrm{mg}$ lipid) for CL-2 and 55\% (8.3 $\mu \mathrm{g} \mathrm{AZI/mg} \mathrm{lipid)} \mathrm{for} \mathrm{DPGL-1.} \mathrm{These} \mathrm{results} \mathrm{are} \mathrm{a} \mathrm{con-}$ sequence of the physicochemical properties of AZI and the liposomal constituents, both affecting encapsulation of the drug. AZI, even in the form of dihydrate, exhibits lipophilic features $(\log P=3.98),{ }^{13}$ therefore it is expected to become accommodated within the liposome bilayers. However, molecular weight of AZI is rather large (Mw 785), ${ }^{13}$ restricting its incorporation within bilayers. The differences in the encapsulation efficiencies observed between the various types of liposomes thus proceed from the effects of the liposomal constituents on AZI incorporation. Generally, liposomes with EPG embedded as a bilayer constituent were able to incorporate significantly larger amounts of the drug (except for DPGL-2) than the corresponding liposomes composed of only neutral phospholipids ( $t$-test, $p<0.05)$. Such results are probably a consequence of the interaction between AZI and the negatively charged EPG inside liposome bilayers, facilitating the encapsulation of AZI. Binding of AZI to negatively charged phospholipids has been confirmed by Van Bambeke et $\mathrm{al}^{36}$. Among the various negatively surface-charged liposomes (CL-1, CL-3, PGL-2 and DPGL-2), both types of elastic liposomes (PGL-2 and DPGL-2) demonstrated significantly higher encapsulation efficiency than CL-1 and CL-3 (ANOVA, $p<0.05$ ). Increased incorporation of AZI in the elastic liposomes was due to the presence of propylene glycol (PGLs, DPGLs) and the monoacyl phospholipid SLPC-80 (DPGLs), which both facilitate solubilization and hence improve encapsulation of AZI. Contrary to the previous findings obtained for deformable liposomes, ${ }^{17,21,23}$ DPGLs exhibited superior drug encapsulation because the presence of monoacyl phosphatidylcholine increased the solubilization of AZI. Namely, monoacyl phospholipid has been proven as a more efficient solubilizer than diacyl phospholipid. ${ }^{37}$ The slightly lower encapsulation of AZI in DPGL-2 than in DPGL-1 could be attributed to the possible interaction/competition of the monoacyl phospholipid and the negatively charged phospholipid inside the bilayers of DPGL-2 for AZI; however, further studies, such as Fourier transform infrared spectroscopy (FT-IR), are necessary to prove the interactions of liposomal bilayer constituents and the drug.

\section{In vitro release studies}

The predictable release of the encapsulated drug from liposomes in medium simulating the in vivo environment is of great significance regarding the use of liposomes as drug delivery nanosystems. As the human vaginal mucosa has a $\mathrm{pH}$ between $\mathrm{pH} 4$ and 5, studies were performed in conditions simulating the vaginal environment, ie, VFS, $\mathrm{pH}$ 4.5. The experiments were also performed in PBS, $\mathrm{pH}$ 7.5 , to mimic cervical conditions, ${ }^{33}$ and to investigate the effect of $\mathrm{pH}$ and vaginal fluid components on AZI release from the liposomes.

The results in Figure 2 demonstrate the prolonged release of AZI from all the liposomes in both tested media relative to the control (AZI solution) (ANOVA, $p<0.05$ ). Overall, the release from all the tested liposomes was slightly increased when the liposomes were dispersed in VFS, $\mathrm{pH} 4.5$, than in PBS, $\mathrm{pH} 7.5$, as a result of the effects of lower $\mathrm{pH}$ and vaginal fluid components on liposome stability. ${ }^{38}$ Despite the used release media, all the liposomes exhibited initial burst release followed by sustained AZI release. Such a release profile might be relevant for facilitating the rapid start of antibiotic activity and ensuring increased local drug concentrations.

Liposome membrane elasticity/rigidity significantly influenced the AZI release profile from the different liposomes under simulated cervicovaginal conditions. Thus, the slowest release, at both $\mathrm{pH} 4.5$ and 7.5, was obtained from the rigid liposomes, ie, CL-2 and CL-3, followed by less rigid CL-1, while AZI release was significantly faster from both types of elastic liposomes. For instance, $70-80 \%$ of the originally encapsulated AZI was released after $6 \mathrm{~h}$ from PGLs and DPGLs under simulated vaginal conditions, compared to $40-45 \%$ with CL-2 and CL-3. Increased AZI release from PGLs, especially from DPGLs, was expected due to the increased membrane permeability caused by propylene glycol (PGLs) in addition to intercalation of the edge activator (SLPC-80) in bilayers of DPGLs. These observations are in accordance with previous reports for PGLs and DPGLs. ${ }^{17,22,39}$ In addition to bilayer elasticity, the highly negative surface charge also increased the release of AZI. Similar behavior of negatively charged liposomes was also observed by Pavelić et $\mathrm{al}^{38}$. However, this effect was negligible in comparison to the impact of membrane elasticity (Figure 2).

\section{Storage stability studies}

The stability of liposomes during storage is an important feature considering their use as drug nanoformulations. As shown in Figure 3, all the liposomes with slightly negative surface charges (CL-2, PGL-1, and DPGL-1) significantly 

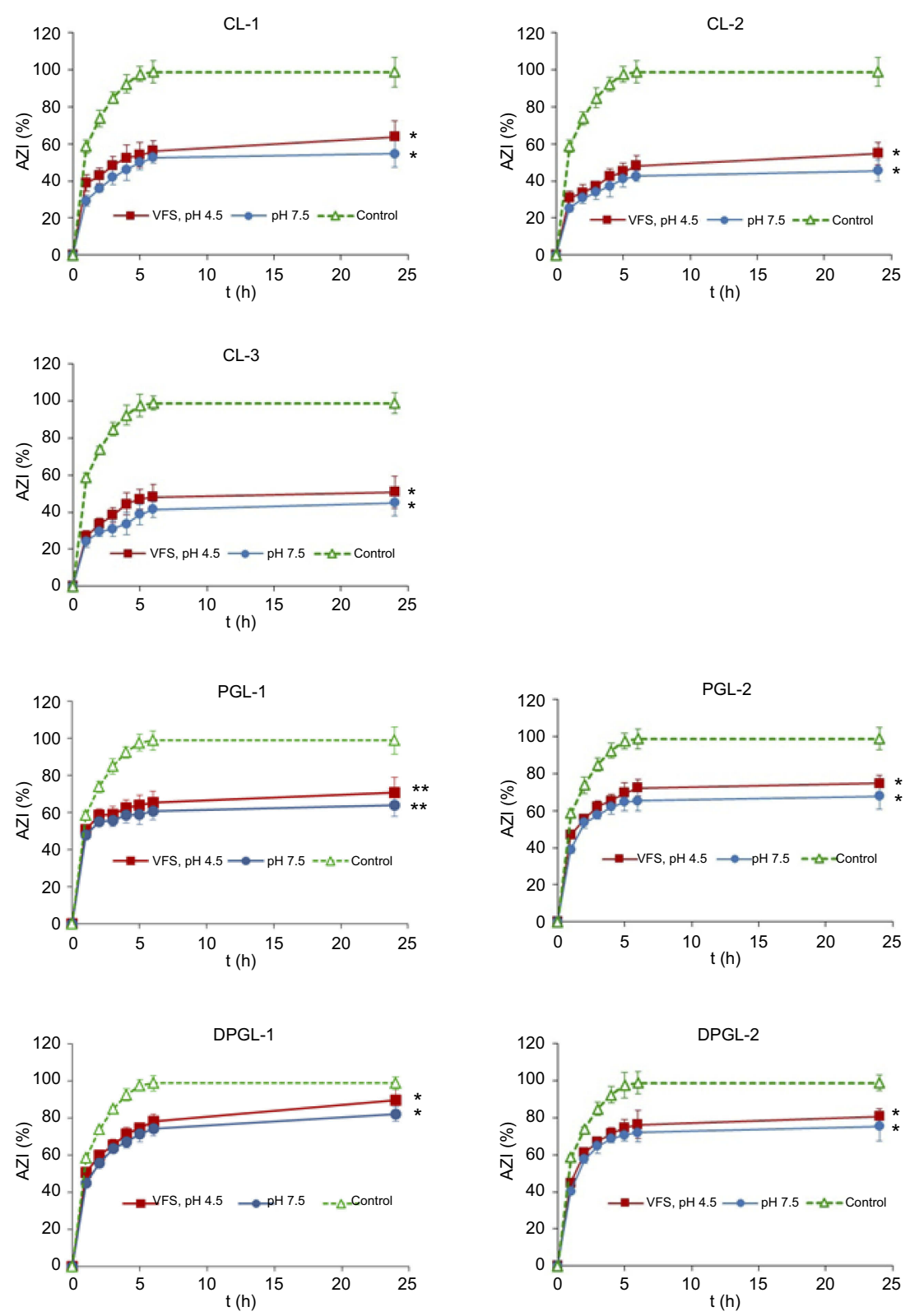

Figure 2 In vitro AZI release from the different types of liposomes.

Notes: The each value represents the mean \pm S.D. $(n=3)$. One-way ANOVA and Tukey's multiple comparison test, with $p<0.05$ set as the minimal level of significance, were applied for comparison of AZI release from the different types of liposomes and control. The control, representing a solution of AZI, was examined at $\mathrm{pH} 7.5$. *Significantly different (ANOVA, $p<0.05$ ) from the control (period I-24 h). **Significantly different (ANOVA, $p<0.05)$ from the control (period $2-24 \mathrm{~h})$.

Abbreviation: $\mathrm{AZI}$, azithromycin.

increased in size (mean diameters and PDIs) during storage in comparison to the corresponding initial values; CL-2 and DPGL-1 increased after 2 months (CL-2 from 221 to $267 \mathrm{~nm}$ and DPGL-1 from 248 to $274 \mathrm{~nm}$ ), while PGL-1 increased after 5 months (from 341 to $372 \mathrm{~nm}$ ) $(t$-test, $p<0.05$ ) (Figure 3A and B). The lower physical stabilities of CL-2 and DPGL-1 were closely associated with their slightly negative surface charges ( -20 and $-18 \mathrm{mV}$, Figure $3 \mathrm{C}$ ). In addition, DPGL-1 was characterized by the significantly pronounced membrane elasticity (15.7, Table 3 ), making their bilayers more permeable and prone to aggregation (fusion). These observations are consistent with the recently reported findings demonstrating the influence of the bilayer elasticity on the reduced physical stability of liposomes incorporating lipophilic drug. ${ }^{17}$ In contrast, the liposomes with highly negative surface charge (zeta potentials between -50 and -60 mV), namely, CL-1, CL-3, PGL-2 and DPGL-2, were physically stable during 7 months of storage (Figure 3C). Similar findings were previously reported by Palac et al, ${ }^{21}$ whereas highly negatively surface charged elastic and rigid 


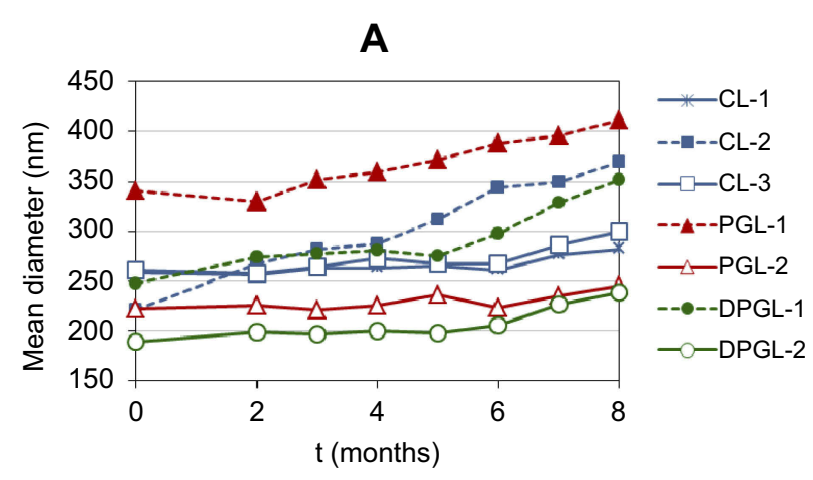

B

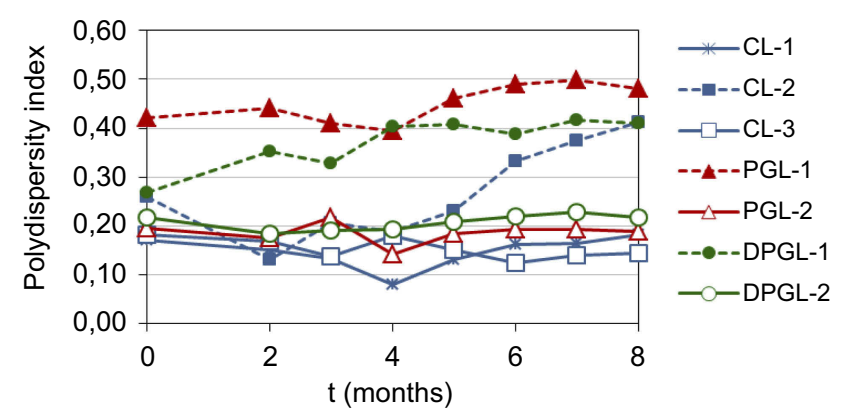

C

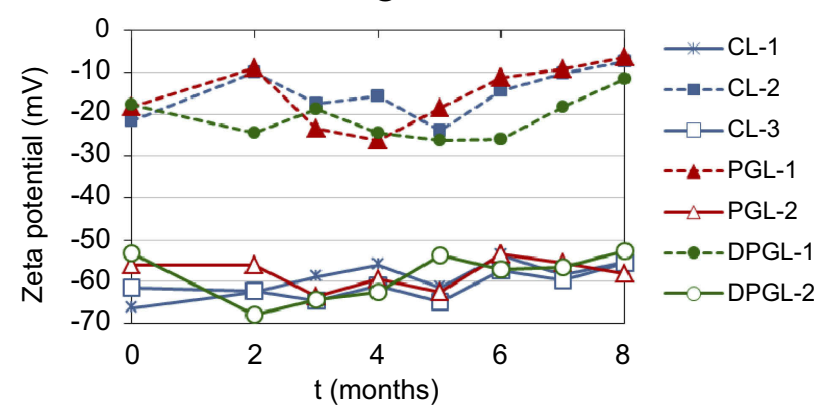

Figure 3 Physical stability of different AZI-liposomes: mean diameters (A), polydispersity indexes (B) and zeta potentials (C).

Notes: The each value represents the mean \pm S.D.; $n=3$ (mean diameter, polydispersity index); $n=5$ (zeta potential). For each type of AZI-liposomes, the values determined during 2-8 months were compared with the corresponding initial values using Student's $t$-test, with $p<0.05$ set as the minimal level of significance. The standard deviations and statistically significant differences are not shown due to the clarity of presentation. Statistically significant increase in mean diameters and polydispersity indexes are determined for CL-2 and DPGL-I (2-8 months) and for PGL-I (5-8 months).

liposomes with encapsulated hydrophilic drug remained physically stable over 10 months of storage at $4^{\circ} \mathrm{C}$.

A comparison of the two different preparations of highly negatively charged CLs (CL-1 and CL-3) showed similar stability profiles. Since the bilayers of CL-3 were significantly more rigid than those of CL-1 (Table 3), it is assumed that CL-3 will be more suitable for further studies due to the lower possibility of drug leakage.

Finally, taking into account the physical characteristics of the liposomes, suitable AZI encapsulation, in vitro AZI release patterns under simulated cervicovaginal conditions and storage stability, CL-3, PGL-2 and DPGL-2 were selected for further investigations. Their composition is schematically depicted by Figure 4. Besides phosphatidylcholine (major phospholipid) and 5\% phosphatidylglycerol (negatively charged lipid) common for all optimized liposomes (CL-3, PGL-2 and DPGL-2), the bilayers of CL-3 were enriched with hydrogenated phosphatidylcholine (SPC-3) contributing to membrane rigidity. On the other hand, elastic liposomes embedded either propylene glycol (PGL-2) or both propylene glycol and monoacyl phospholipid (DPGL-2) in their bilayers, decreasing bilayers' compactness, while increasing its elasticity.

\section{Ex vivo permeation studies}

Localized antimicrobial therapy has been used to treat vaginal infections. However, its efficiency is frequently reduced because of several factors. For instance, possible systemic drug absorption and drug teratogenicity (metronidazole) can take place, and inadequate local drug concentrations may be achieved, along with the possible development of antibiotic-resistant strains, the formation of biofilms and the failure of the drugs to reach the desired site of action. These possibilities make oral administration of high doses of antibiotics necessary, which may lead to an increased incidence of undesired drug side effects, patient inconvenience and an enhanced risk of antibiotic resistance development. ${ }^{40}$

The use of liposomes represents a suitable approach to achieve effective localized vaginal therapy. Manipulation of the physical characteristics of liposomes (bilayer elasticity/rigidity, surface coating) enables the delivery of encapsulated drugs to the site of action. ${ }^{10}$ Therefore, to more closely resemble the applicability of AZI-liposomes for the treatment of vaginal infections, ex vivo permeation studies were performed.

The results presented in Figure 5 refer to AZI levels determined in the acceptor chamber (penetrated AZI), within vaginal tissue, and on the vaginal surface (nonpenetrated AZI) after $24 \mathrm{~h}$ of testing. It was not possible to detect the presence of AZI in the acceptor chamber during the first $6 \mathrm{~h}$ of testing since the drug content was under the limit of detection. The only exception was the control (free AZI), for which a negligible amount of the drug was detected after $5 \mathrm{~h}$.

The performed ex vivo studies undoubtedly demonstrate the potential of AZI-liposomes in localized vaginal therapy (Figure 5). All tested liposomes enabled accumulation of the drug on the vaginal surface (17-24\%) and mostly inside 


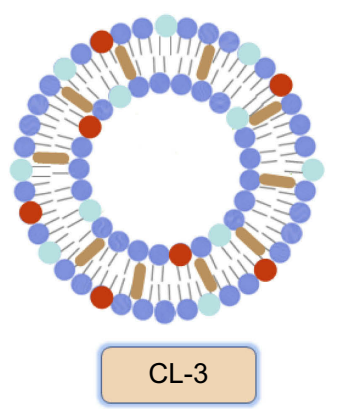

Conventional liposomes

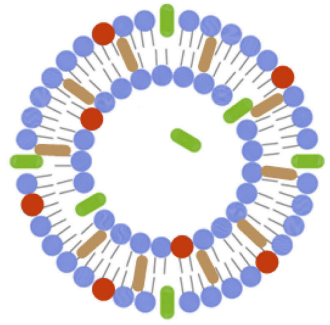

PGL-2

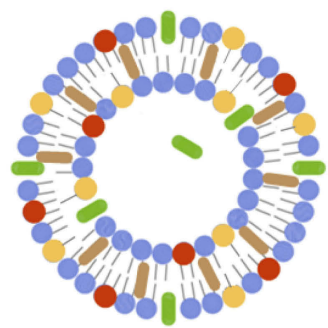

DPGL-2

$\begin{array}{ll}\text { Phosphatidylcholine } & \text { Monoacyl Phosphatidylcholine } \\ \text { १ydrogenated Phosphatidylcholine } & \text { Propylene glycol } \\ 9 \text { Phosphatidylglycerol } & \text { Azithromycin }\end{array}$

Figure 4 Schematic drawing of the AZI-liposomes explored for ex vivo vaginal deposition/penetration, in vitro antibacterial activity and biocompatibility studies.

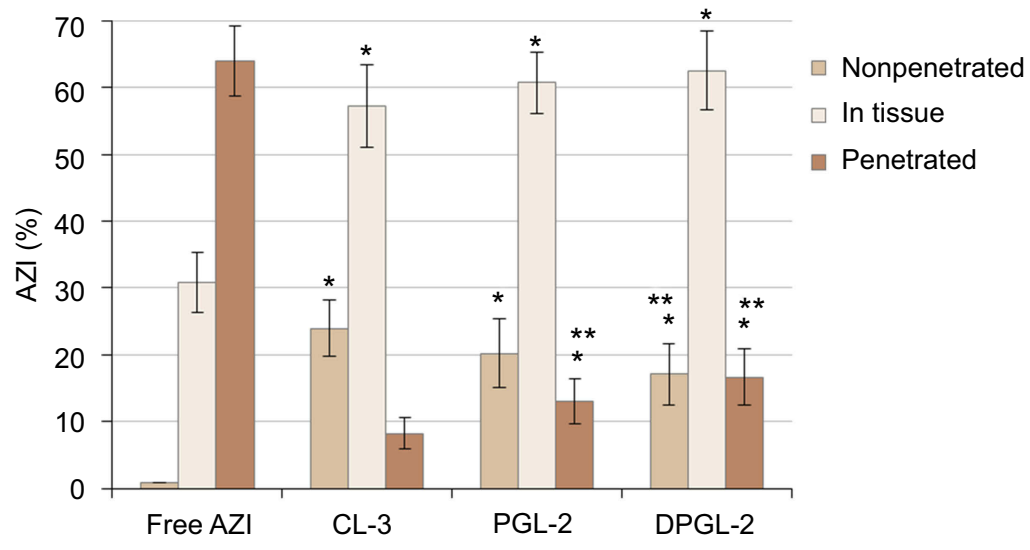

Figure 5 Ex vivo deposition/penetration of AZI into/through porcine vaginal tissue.

Notes: The each value represents the mean \pm S.D. $(n=3)$. One-way ANOVA and Tukey's multiple comparison test, with $p<0.05$ set as the minimal level of significance, were applied for comparison of the levels of nonpenetrated, penetrated and AZI retained in the tissue, from different types of AZI-liposomes and control. *Significantly different (ANOVA, $p<0.05$ ) from free AZI (control). **Significantly different (ANOVA, $p<0.05$ ) from CL-3.

Abbreviation: AZI, azithromycin.

vaginal tissue (57-63\%), generating higher local concentrations of AZI and reducing its undesired penetration to the systemic circulation compared to the control (free AZI). Increased retention of the drug inside the vagina could be relevant for the treatment of recurrent bacterial vaginosis and biofilm-related infections. Comparison of the different types of liposomes demonstrated the highest penetration ability for DPGL-2, followed by PGL-2 and CL-3 (Figure 5). These findings are consistent with the bilayer properties of liposomes (Table 3) influencing AZI deposition/penetration in vaginal tissue. For instance, CL-3 had rigid bilayers facilitating retention of the AZI on/within the vaginal tissue. In contrast, the highest AZI penetration was observed with DPGL-2 (Figure 5), which is in accordance with its very elastic bilayers, facilitating increased drug penetration into/ through the vaginal tissue.

The physicochemical properties of liposomes significantly contribute to the vaginal deposition/penetration abilities of the encapsulated drug. Thus, mucoadhesive, chitosan-coated liposomes enabled the localization of clotrimazole on the vaginal surface, ${ }^{41}$ while non-mucoadhesive, ie, mucus-penetrating, PEGylated liposomes demonstrated increased interferon alpha penetration into/through the vaginal mucosa, ${ }^{42}$ in comparison to controls (solutions of the free drugs at the same 
concentrations present in the tested liposomes). In a recent study, positively charged liposomes with rigid bilayers enabled localization of AZI on the skin surface due to strong interactions with the negatively charged skin cells. ${ }^{17}$

\section{In vitro antibacterial activity}

The optimized AZI-liposomes (CL-3, PGL-2 and DPGL2) were investigated for their in vitro antibacterial activity against several biofilm-forming and planktonic E. coli strains (Tables 5 and 6) and against intracellular C. trachomatis (Figure 6).

The results for planktonic $E$. coli are expressed as $\mathrm{MIC}_{50}$ values, while those obtained for the pre-exposure mode of the biofilm assay are presented as $\mathrm{IC}_{50}$ values (Table 5).

As shown in Table 5, the antibacterial activity of all the AZI samples (both liposomally encapsulated and free AZI) varied among the strains. In general, E. coli $\mathrm{K}-12$ was the most sensitive to AZI, followed by E. coli ATCC 700928 and ATCC 8739. All the AZI-liposomes were more effective than the free AZI against E. coli ATCC 700928, with $\mathrm{MIC}_{50}$ values approximately 3-fold lower than the $\mathrm{MIC}_{50}$ of the free AZI. A comparison of the activities achieved by the different types of liposomes toward E. coli K-12 demonstrated the similar efficacy of CL-3 and PGL-2, while the activity of DPGL-2 was significantly lower (ANOVA, $p<0.05$ ). Regarding E. coli ATCC 8739, all of the AZI-liposomes demonstrated activity similar to that of the free antibiotic; CL-3 exhibited slightly lower activity, but this activity was not significantly different from that of free AZI ( $t$-test; $p>0.05)$.

Similarly, the observed anti-biofilm activity of the AZIliposomes varied between strains. For example, CL-3 was the most effective against E. coli ATCC 700928 and K-12, exhibiting $\mathrm{IC}_{50}$ values almost 8-fold lower than those of the free antibiotic. PGL-2 and DPGL-2 demonstrated lower anti-biofilm activity than CL-3 (ANOVA, $p<0.05$ ), but this activity was still significantly stronger than that of the free AZI ( $t$-test, $p<0.05)$. PGL-2 inhibited E. coli ATCC 8739 biofilm formation with an $\mathrm{IC}_{50}$ of $6.36 \mu \mathrm{g} / \mathrm{ml}$, which was twice as low as the $\mathrm{IC}_{50}$ of the free AZI (Table 5). By contrast, CL-3 and DPGL-2 displayed weaker anti-biofilm activity (Table 5).

The observed variability in the activity of the different types of AZI-liposomes tested might be a consequence of the bilayer properties of the tested liposomes affecting AZI release and the susceptibility of the different $E$. coli strains to AZI. CL-3 contained hydrogenated phospholipids in the bilayers, contributing to a higher phase transition temperature, ${ }^{43}$

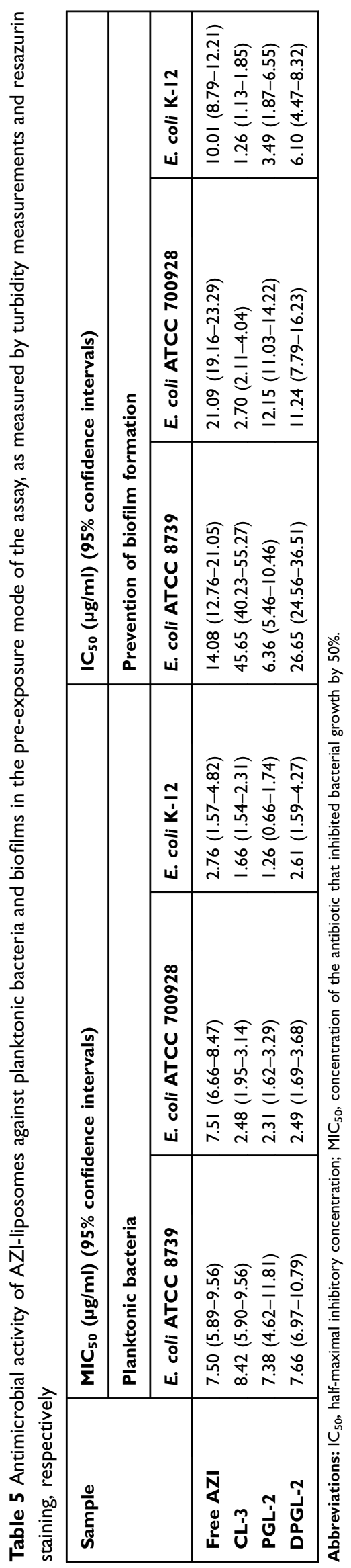


Table 6 Eradication of $E$. coli biofilms at the highest tested concentration $(78.50 \mu \mathrm{g} / \mathrm{ml})$ in the postexposure mode of the assay, as measured using a resazurin staining assay

\begin{tabular}{|l|l|l|l|}
\hline \multirow{2}{*}{ Sample } & \multicolumn{2}{|l|}{ Inhibition of biofilm viability (\%) } \\
\cline { 2 - 4 } & E. coli ATCC 8739 & E. coli ATCC 700928 & E. coli K-I2 \\
\hline Free AZI & $17.49 \pm I .54$ & $26.44 \pm 7.14$ & $26.88 \pm 6.00$ \\
CL-3 & $16.44 \pm 2.31$ & $25.15 \pm 4.83$ & $26.95 \pm 3.06$ \\
PGL-2 & $26.94 \pm I .86$ & $27.15 \pm 5.09$ & $30.31 \pm 1.55$ \\
DPGL-2 & $25.24 \pm \mid 4.01$ & $45.67 \pm 7.14^{*}$ & $38.08 \pm \mid 3.74^{* *}$ \\
\hline
\end{tabular}

Notes: The values indicate the mean \pm S.D. $(n=4)$. *Statistically significant compared to free AZI, PGL-2 and CL-3 (ANOVA, $p<0.05)$. **Statistically significant compared to free AZI, PGL-2 and CL-3 (ANOVA, $p<0.05$ ).

Abbreviation: $A Z I$, azithromycin.

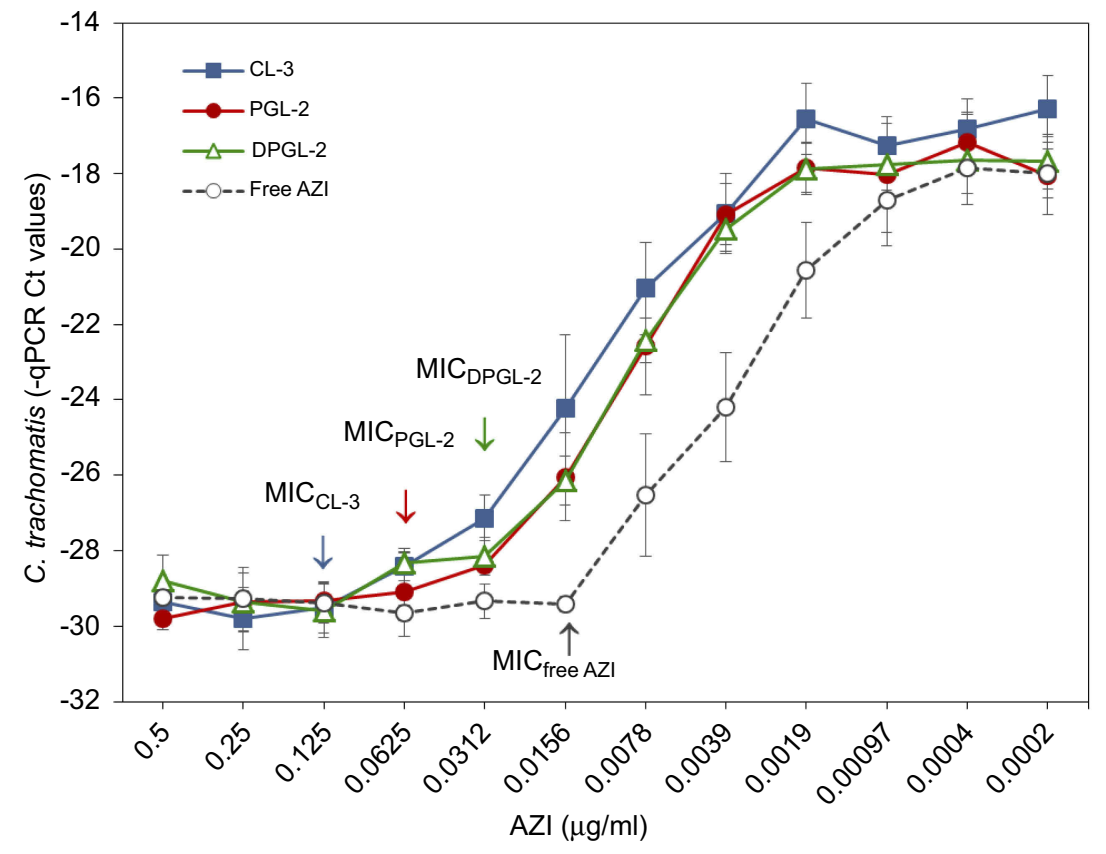

Figure 6 In vitro activities of AZI-liposomes against C. trachomatis presented as MIC values.

Notes: $C$. trachomatis (MOI 0.2)-infected HeLa cells were cultured in the presence of $0.5-0.0002 \mu g / m l$ free or liposomally loaded AZI (CL-3, PGL-2 and DPGL-2). C. trachomatis genome concentrations were measured at $48 \mathrm{~h}$ post infection by direct qPCR. Data are presented as the mean $C t$ levels \pm S.D. ( $n=3$ ).

Abbreviations: AZI, azithromycin; $\mathrm{Ct}$, cycle threshold; MIC, minimum inhibitory concentration; qPCR, quantitative polymerase chain reaction.

allowing slower AZI release, which could be relevant for preventing biofilm formation by E. coli ATCC 700928 and K-12.

To examine the potential of AZI-liposomes to eradicate preformed E. coli biofilms, the biofilms were exposed to AZI samples (both liposomally encapsulated and the free drug).

As shown in Table 6, the strongest inhibition of the $E$. coli biofilms was achieved with DPGL-2, even though this formulation showed lower activities than CL-3 and PGL-2 against planktonic E. coli and toward the suppression of biofilm formation (pre-exposure mode). DPGL-2 inhibited E. coli ATCC 700928 biofilm viability by $46 \%$ compared with the $27 \%$ and $26 \%$ inhibition obtained with PGL-2 and the free AZI, respectively. However, its anti-biofilm activity varied between strains. The strongest inhibition (ANOVA, $p<0.05$ ) was obtained against $E$. coli ATCC 700928 biofilms, followed by $E$. coli $\mathrm{K}-12$ and $E$. coli ATCC 8739 biofilms (Table 6). When tested against E. coli ATCC 8739, PGL-2 was the most active among the different AZI-liposomes (27\% inhibition), but it was not significantly more active versus DPGL-2 (25\% inhibition, ANOVA, $p>0.05)$. However, PGL-2 displayed significantly enhanced activity compared to the free antibiotic (17\%), while the anti-biofilm activity of CL-3 was similar to that of the free drug (Table 6).

Overall, anti-biofilm activity (Table 6) was lower than in the pre-exposure mode of the assay (Table 5). These findings are in agreement with the well-known fact that eradication of bacterial biofilms is difficult to achieve, even with 
the application of very high concentrations of antibiotics. ${ }^{44}$ The enhanced activities of elastic liposomes, particularly DPGL-2 against E. coli biofilms, were probably a consequence of the bilayer properties of the elastic liposomes, which permit their enhanced penetration into the formed biofilms. Additionally, the presence of monoacyl phosphatidylcholine in DPGL-2 has been shown to increase antibiofilm activity.

A limited number of studies reported on the efficacy of liposomal antibiotics against planktonic $E$. coli, but none examined activity against $E$. coli biofilms. According to a literature search, only so-called fusogenic liposomes encapsulating tobramycin or vancomycin have been examined against planktonic E. coli. ${ }^{45,46}$ Their superior activities in comparison to the free antibiotics were considered a result of the confirmed fusion of the vesicles composed of dioleoyl phosphatidylethanolamine, dipalmitoyl phosphatidylcholine and cholesteryl hemisuccinate (4:2:4, molar ratio) with $E$. coli, allowing release of the encapsulated vancomycin in the cytoplasm of E. coli. ${ }^{46}$

The obtained data for in vitro antibacterial activity (Tables 5 and 6) are important regarding the potential use of AZI-liposomes in localized vaginal therapy for $E$. coli infections. CL-3 and PGL-2 would have potential for the treatment of superficial infections caused by planktonic E. coli and the prevention of complicated infections, while DPGL-2 seems the most promising among the tested liposomes for the treatment of biofilm-related infections. The AZI-liposomes with elastic bilayers exhibited enhanced antibacterial activities against Staphylococcus aureus and MRSA clinical isolates, ${ }^{17}$ which is of great relevance considering the possible use of AZI-liposomes for the treatment of aerobic vaginitis caused by both E. coli and $S$. aureus. Cervicovaginal infections caused by $S$. aureus have also been reported to increase the risk of fetal complications in pregnant women, similar to E. coli. ${ }^{47}$ In the present study, all AZI-liposomes were negatively charged, which could be significant regarding the biocompatibility issue. Interestingly, the negative surface charge has also been shown to influence the antimicrobial efficacy of AZIliposomes toward $M$. avium, as demonstrated by $\mathrm{Oh}$ et $\mathrm{al}^{16}$. Since AZI is a potent broad-spectrum antibiotic, we assume that AZI-liposomes could be suitable for the treatment of recurrent bacterial vaginosis caused by bacteria other than E. coli and S. aureus, based on the reported efficacy of AZI against Gardnerella vaginalis, Atopobium vaginae, Bacteroides spp., Mobiluncus spp. and Mycoplasma spp. ${ }^{48,49}$ Moreover, high doses of AZI are recommended as the first choice of therapy for the treatment of $A$. vaginae and $G$. vaginalis bacterial vaginosis to prevent preterm birth. ${ }^{50}$ Therefore, it seems reasonable to broaden the possible applicability of AZI-liposomes for the treatment of recurrent bacterial vaginosis; however, the efficacy of the developed liposomes has to be investigated to prove these assumptions.

In addition to $E$. coli, the AZI-liposomes were also tested against intracellular $C$. trachomatis. A direct qPCR method was applied to determine the MICs of the free AZI and the liposomally loaded AZI (CL-3, PGL-2, and DPGL-2). C. trachomatis (MOI 0.2)-infected HeLa cells were cultured in the presence of $0.5-0.0002 \mu \mathrm{g} / \mathrm{ml} \mathrm{AZI}$ samples, and the dose-response curves were evaluated $48 \mathrm{~h}$ post infection (Figure 6). The MIC values were identified by statistical comparison of the bacterial DNA concentrations ( $\mathrm{Ct}$ values) measured in the presence of a given AZI concentration with the $\mathrm{Ct}$ values of bacterial DNA in the presence of the highest AZI concentration. The $\mathrm{Ct}$ levels measured in the presence of the highest antibiotic concentrations were considered as the genome concentration of the inoculum. The MIC value was defined as the lowest AZI concentration, where the $\mathrm{Ct}$ values were not significantly different from the inoculum. ${ }^{28}$

Among the different types of AZI-liposomes tested against C. trachomatis, DPGL-2 was the most effective, with MIC value of $0.0312 \mu \mathrm{g} / \mathrm{ml}$, followed by PGL-2 $(0.0625 \mu \mathrm{g} / \mathrm{ml})$ and CL-3 $(0.125 \mu \mathrm{g} / \mathrm{ml})$ (Figure 6). These results correlate with the bilayer elasticity/rigidity of the examined liposomes (Table 4), leading to more rapid AZI release from DPGL-2 than CL-3 (Figure 2). The lower efficacy of all the AZI-liposomes than the control (free AZI) could be a consequence of the fact that C. trachomatis is an intracellular pathogen and that lipophilic AZI in solution form is more easily taken up by the cells than liposomes, ${ }^{51,52}$ which was also confirmed by ex vivo penetration studies (Figure 5), showing higher penetration of free AZI through the vaginal tissue. On the other hand, the surface charge of the liposomes also plays an important role in the internalization of the encapsulated drug. Generally, positively surface-charged (cationic) liposomes allow fusion with negatively surface-charged epithelial cells, enabling efficient intracellular drug delivery. ${ }^{8}$ Since all the tested liposomes in this study were negatively surface charged, it is assumed that they exhibited lower affinity toward HeLa cells than the free drug, which is supported by Sangaré and collaborators, ${ }^{53}$ who demonstrated that cationic liposomes encapsulating doxycycline were 2-fold more effective than free doxycycline against C. trachomatis in vitro. Therefore, the efficacy of the 
positively surface-charged AZI-liposomes against C. trachomatis would be worthy to evaluate in future investigations.

Considering that the impact of antimicrobials on chlamydial growth was evaluated by direct qPCR with unpurified cell lysates, we measured the impact of the unpurified cell lysates on the performance of qPCR (Figure 7). Namely, it should be excluded whether the tested samples (AZI-liposomes and free AZI) had a direct inhibitory or stimulatory effect on the qPCR, influencing the dose-response curves of the AZI samples (AZI-liposomes and free AZI). To test this possible effect, Chlamydia-specific qPCRs were performed in the presence and absence of AZI (AZI-liposomes and free AZI). For that purpose, C. trachomatis-infected HeLa cell lysates were mixed with uninfected but AZI-treated (AZI-liposomes or free AZI) HeLa cell lysates. The final concentration of AZI was the highest concentration applied in the chlamydial growth inhibition experiments. Before cell lysis, similar to the dose-response curve experiments, the medium was removed, and the cells were washed with PBS. As a control, C. trachomatis-infected HeLa cell lysates were mixed with uninfected and AZI untreated HeLa cell lysates. If the AZI samples had no impact on the qPCR, then the AZI-containing samples had $\mathrm{Ct}$ levels similar to the $\mathrm{Ct}$ levels of the control.

The results presented in Figure 7 show that the detected chlamydial genome concentrations ( $\mathrm{Ct}$ levels) of AZI samples (liposomes and the free drug) and the sample without AZI (control) were not significantly different $(t$ test; $p>0.05)$. Consequently, qPCR measurement of

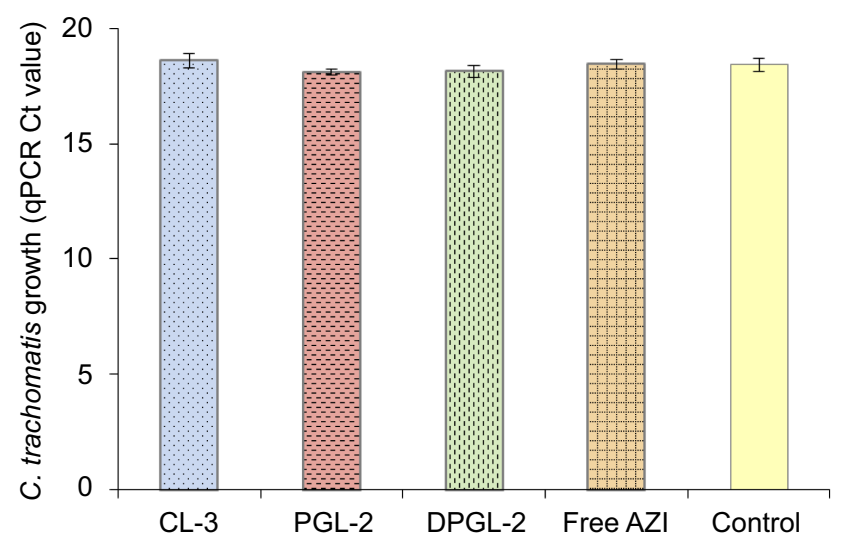

Figure 7 Estimation of the impact of AZI-liposomes and free AZI on qPCR. Notes: Chlamydia-specific qPCRs were performed in the presence of AZI-liposomes and free $\mathrm{AZI}$ at the highest applied concentrations that were used in the dose-response curve measurement experiments. One-way ANOVA and Tukey's multiple comparison test, with $p<0.05$ set as the minimal level of significance, were applied for comparison of qPCR Ct levels of CL-3, PGL-2, DPGL-2 and free AZI containing samples and control. No significant differences were found $(p>0.05)$. Data are presented as the mean Ct value \pm S.D. $(n=3)$.

Abbreviations: $\mathrm{AZI}$, azithromycin; $\mathrm{Ct}$, cycle threshold; $\mathrm{QPCR}$, quantitative polymerase chain reaction. chlamydial growth inhibition was not influenced by the applied compounds.

\section{Biocompatibility studies}

The biocompatibility of AZI-liposomes (CL-3, PGL-2 and DPGL-2) was investigated in vitro to determine the possible cytotoxic effects of the liposome constituents and AZI toward human cervical cells (HeLa cells). It was reported that AZI can synergistically enhance the antiproliferative activity of vincristine in cancer cells. ${ }^{54}$ Furthermore, recent findings demonstrated that free AZI was cytotoxic to human fibroblasts at a concentration of $16 \mu \mathrm{g} / \mathrm{ml}(49 \%$ viability) and human keratinocytes at $64 \mu \mathrm{g}$ AZI $/ \mathrm{ml}(55 \%$ viability), while entrapment of AZI in liposomes significantly decreased skin cell cytotoxicity. ${ }^{17}$

The performed studies showed that all AZI-liposomes were compatible with the HeLa cells (Figure 8A). Even at the highest tested concentration $(39.25 \mu \mathrm{g} / \mathrm{ml})$, the viability of the cells remained above $70 \%$, which is considered a noncytotoxic threshold. ${ }^{55}$ Comparison of the different types of AZI-liposomes proved CL-3 to be the most compatible, while the HeLa cells were more sensitive to both types of elastic liposomes, particularly DPGL-2, at the highest tested AZI concentration, which covered and exceeded the $\mathrm{MIC}_{50}$ and $\mathrm{IC}_{50}$ values for $E$. coli and significantly surpassed the MIC values for $C$. trachomatis.

Evaluation of the empty liposomes (without encapsulated AZI) at concentrations that corresponded to AZI-liposomes proved the biocompatibility of all tested liposomes (Figure 8B). Therefore, the slightly increased sensitivity of the HeLa cells toward elastic AZI-liposomes (PGL-2, DPGL-2) (Figure 8A) is thought to be a result of the increased AZI release from the elastic liposomes (Figure 2), facilitated by the presence of $10 \%$ propylene glycol in dispersions of PGL-2 and DPGL-2, in addition to $15 \%$ monoacyl phosphatidylcholine inside the bilayers of DPGL-2 (Table 1). These assumptions are supported by recent findings demonstrating the sensitivity of human keratinocytes and fibroblasts to deformable liposomes, which was also affected by increased drug release and the presence of an edge activator (sodium deoxycholate). ${ }^{17}$

Although monoacyl phospholipids (lysophospholipids) have been proven to contribute to cell cytotoxicity at concentrations $>50 \mu \mathrm{g} / \mathrm{ml},{ }^{56}$ in this study the concentration of monoacyl phosphatidylcholine in DPGL-2 was significantly lower ( $39 \mu \mathrm{g} / \mathrm{ml}$ at the highest tested concentration), and the exposure time was $24 \mathrm{~h}$ in comparison to $72 \mathrm{~h} .{ }^{56}$ 

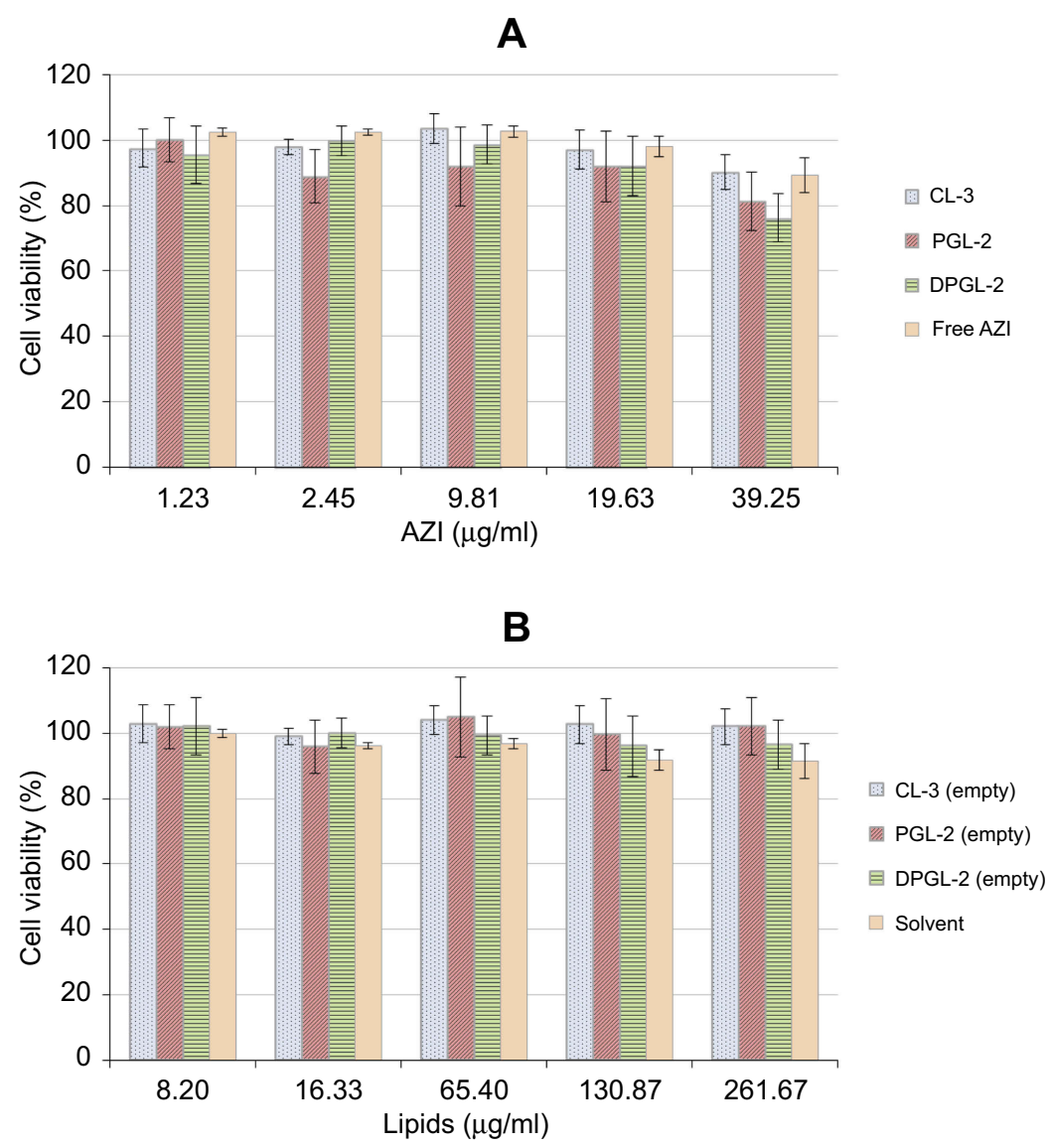

Figure 8 Viability of HeLa cells after incubation with different AZI-liposomes or free AZI (A) and with the corresponding empty liposomes (without AZI) or the solvent used to dissolve free AZI (B).

Note: The presented values are the mean \pm S.D. $(n=3)$.

Abbreviation: AZI, azithromycin.

Hence, monoacyl phosphatidylcholine did not contribute to the cytotoxic effects of DPGL-2 under the applied conditions, even though there was a general trend of lower cell viability at higher AZI concentrations in DPGL-2 (Figure 8A). However, the possible cytotoxicity of DPGL-2 might be expected at higher concentrations of monoacyl phospholipid and with longer exposure of DPGL-2 to cervical cells (72 h).

The biocompatibility of the liposomes examined in this research could also be a consequence of their negative surface charge. Namely, cationic liposomes incorporating dimethyldioctadecyl ammonium bromide applied at higher concentrations significantly decreased the viability of A549 human lung cells and fibroblasts, ${ }^{17,57}$ in comparison to the corresponding negatively charged liposomes.

\section{Conclusion}

The results of all the performed studies indicate that targeting cervicovaginal infections requires careful tailoring of the liposomal characteristics to yield stable liposomes that are biocompatible with cervical cells, allowing sustained AZI release and localization within vaginal tissue while enhancing antibacterial activity. The selection of AZI-liposomes should be based on the targeted bacterial strain. CL-3 would be optimal for preventing E. coli 700928 and K12 biofilms, while PGL-2 would be optimal for inhibiting the formation of $E$. coli 8739 biofilms. DPGL-2 exhibited the strongest potential for the eradication of $E$. coli biofilms and was the most promising among the examined liposomes toward $C$. trachomatis. Further formulation development should focus on ensuring the prolonged residence time of AZI-liposomes in the vaginal cavity by inclusion in a secondary vehicle, such as a mucoadhesive hydrogel.

\section{Abbreviation list}

AZI, azithromycin; CFU, colony forming units; CLs, conventional liposomes; Ct, cycle threshold; DPGLs, deformable propylene glycol liposomes; E, degree of liposome membrane 
elasticity; EPC, egg phosphatidylcholine, EPG, egg phosphatidylglycerol sodium salt; $\mathrm{IC}_{50}$, half-maximal inhibitory concentration; J, mass of extruded liposomes in elasticity measurements; LB, Luria-Bertani; MIC, minimum inhibitory concentration; $\mathrm{MIC}_{50}$, concentration of the antibiotic that inhibited bacterial growth by $50 \%$; MRSA, methicillin-resistant Staphylococcus aureus; PBS, phosphate-buffered saline; PCS, photon correlation spectroscopy; PDI, polydispersity index; PGLs, propylene glycol liposomes; qPCR, quantitative polymerase chain reaction; $r_{p}$, pore size of the membrane in elasticity measurements; $r_{v}$, average diameter of the liposomes after passage through a defined membrane in elasticity measurements; SLPC-80, monoacyl phosphatidylcholine from soybean; SPC-3, hydrogenated soybean phosphatidylcholine; VFS, vaginal fluid simulant.

\section{Acknowledgments}

This research was financed by the Phospholipid Research Centre (Heidelberg, Germany). The authors are very thankful to Lipoid (Ludwigshafen, Germany) and PLIVA (Zagreb, Croatia) for the donation of phospholipids and AZI, respectively. We would also like to appreciate the contribution of the Jane \& Aatos Erkko Foundation to A.F.

\section{Disclosure}

Dr Adyary Fallarero is currently employed by Thermo Fisher Scientific in Vantaa, Finland. The authors report no other conflicts of interest in this work.

\section{References}

1. Sáez-López E, Guiral E, Fernández-Orth D, et al. Vaginal versus obstetric infection Escherichia coli isolates among pregnant women: antimicrobial resistance and genetic virulence profile. PLoS One. 2016;11(1):e0146531. doi:10.1371/journal.pone.0146531

2. Adachi K, Klausner JD, Bristow CC, et al. Chlamydia and gonorrhea in HIV-infected pregnant women and infant HIV transmission. Sex Transm Dis. 2015;42(10):554-565. doi:10.1097/OLQ.0000000000000340

3. Centers for Disease Control and Prevention (CDC), Sexually Transmitted Diseases Treatment Guidelines. 2015. Available from: https://www.cdc. gov/std/tg2015/chlamydia.htm. Accessed January 15, 2019.

4. Coyle EA, Prince RA. Urinary tract infections and prostatitis. In: Wells BG, DiPiro JT, Schwinghammer TL, DiPiro CV, editors. Pharmacotherapy Handbook. London: McGraw Hill Medical; 2009:544-555.

5. Abed N, Couvreur P. Nanocarriers for antibiotics: a promising solution to treat intracellular bacterial infections. Int $J$ Antimicrob Agents. 2014;43(6):485-496. doi:10.1016/j.ijantimicag.2014.02.009

6. Škalko-Basnet N, Vanić Ž. Lipid-based nanopharmaceuticals in antimicrobial therapy. In: Boukherroub R, Szunerits S, Drider D, editors. Functionalized Nanomaterials for the Management of Microbial Infection, A Strategy to Address Microbial Drug Resistance. Amsterdam: Elsevier; 2017:111-152.
7. Rukavina Z, Vanić Ž. Current trends in development of liposomes for targeting bacterial biofilms. Pharmaceutics. 2016;8(2):18. doi: $10.3390 /$ pharmaceutics 8020018

8. Allen TM, Cullis PR. Liposomal drug delivery systems: from concept to clinical applications. Adv Drug Del Rev. 2013;65(1):36-48. doi:10.1016/j.addr.2012.09.037

9. Sala M, Diab R, Elaissari A, Fessi H. Lipid nanocarriers as skin drug delivery systems: properties, mechanisms of skin interactions and medical applications. Int $J$ Pharm. 2018;535(1-2):1-17. doi:10.1016/j.jpharm.2017.10.046

10. Vanić Ž, Škalko-Basnet N. Nanopharmaceuticals for improved topical vaginal therapy: can they deliver? Eur J Pharm Sci. 2013;50 (1):29-41. doi:10.1016/j.ejps.2013.04.035

11. Peters DH, Friedel HA, McTavish D. Azithromycin: a review of its antimicrobial activity, pharmacokinetic properties and clinical efficacy. Drugs. 1992;44(5):750-799. doi:10.2165/00003495-199244050-00007

12. Wu EC, Kowalski RP, Romanowski EG, Mah FS, Gordon YJ, Shanks RMQ. AzaSite ${ }^{\circledR}$ inhibits Staphylococcus aureus and coagulase-negative Staphylococcus biofilm formation in vitro. J Ocul Pharmacol Ther. 2010;26(6):557-562. doi:10.1089/jop.2009.0059

13. Mandić Z. Voltammetric study of the partitioning of macrolide antibiotics at the water/nitrobenzene interface: relationship to the pharmacokinetic profiling of macrolides. Admet Dmpk. 2014;2(3):168178. doi:10.5599/admet.2.3.46

14. Imamura Y, Higashiyama Y, Tomono K, et al. Azithromycin exhibits bactericidal effects on Pseudomonas aeruginosa through interaction with the outer membrane. Antimicrob Agents Chemother. 2005;49 (4):1377-1380. doi:10.1128/AAC.49.2.612-618.2005

15. Liu X, Li Z, Wang X, et al. Novel antimicrobial peptide-modified azithromycin-loaded liposomes against methicillin resistant Staphylococcus aureus. Int J Nanomed. 2016;11:6781-6794. doi:10.2147/IJN.S107107

16. Oh YK, Nix DE, Straubinger RM. Formulation and efficacy of liposome-encapsulated antibiotics for therapy of intracellular Mycobacterium avium infection. Antimicrob Agents Chemother. 1995;39(9):2104-2111. doi:10.1128/aac.39.9.1920

17. Rukavina Z, Šegvić Klarić M, Filipović-Grčić J, Lovrić J, Vanić Ž. Azithromycin-loaded liposomes for enhanced topical treatment of methicillin-resistant Staphylococcus aureus (MRSA) infections. Int $J$ Pharm. 2018;553(1-2):109-119. doi:10.1016/j.ijpharm.2018.10.024

18. Solleti VS, Alhariri M, Halwani M, Omri A. Antimicrobial properties of liposomal azithromycin for Pseudomonas infections in cystic fibrosis patients. J Antimicrob Chemother. 2015;70(3):784-796. doi:10.1093/jac/dku445

19. Owen DH, Katz DF. A vaginal fluid simulant. Contraception. 1999;59(2):91-95. doi:10.1016/S0010-7824(99)00010-4

20. Pavelić Ž, Škalko-Basnet N, Jalšenjak I. Characterisation and in vitro evaluation of bioadhesive liposome gels for local therapy of vaginitis. Int J Pharm. 2005;301(1-2):140-148. doi:10.1016/j.jpharm.2005.05.022

21. Palac Z, Engesland A, Flaten GE, Škalko-Basnet N, Filipović-Grčić J, Vanić Ž. Liposomes for (trans)dermal drug delivery: the skinPVPA as a novel in vitro stratum corneum model in formulation development. J Liposome Res. 2014;24(4):313-322. doi:10.3109/ 08982104.2014.899368

22. Vanić Ž, Hurler J, Ferderber K, Golja Gašparović P, Škalko-Basnet N, Filipović-Grčić J. Novel vaginal drug delivery system: deformable propylene glycol liposomes-in-hydrogel. J Liposome Res. 2014;24 (1):27-36. doi:10.3109/08982104.2013.826242

23. Vanić Ž, Hafner A, Bego M, Škalko-Basnet N. Characterization of various deformable liposomes with metronidazole. Drug Dev Ind Pharm. 2013;39(3):481-488. doi:10.3109/03639045.2012.670247

24. Manner S, Vahermo M, Skogman ME, et al. New derivatives of dehydroabietic acid target planktonic and biofilm bacteria in Staphylococcus aureus and effectively disrupt bacterial membrane integrity. Eur J Med Chem. 2015;102:68-79. doi:10.1016/j.ejmech.2015.07.038 
25. Mosolygó T, Spengler G, Endrész V, Laczi K, Perei K, Burián K. IL$17 \mathrm{E}$ production is elevated in the lungs of $\mathrm{Balb} / \mathrm{c}$ mice in the later stages of Chlamydia muridarum infection and re-infection. In Vivo. 2013;27(6):787-792.

26. Sabet SF, Simmons J, Caldwell HD. Enhancement of Chlamydia trachomatis infectious progeny by cultivation of HeLa 229 cells treated with DEAE-dextran and cycloheximide. J Clin Microbiol. 1984;20(2):217-222.

27. Bogdanov A, Janovák L, Lantos I, et al. Nonactivated titaniumdioxide nanoparticles promote the growth of Chlamydia trachomatis and decrease the antimicrobial activity of silver nanoparticles. $J$ Appl Microbiol. 2017;123:1335-1345. doi:10.1111/jam. 13560

28. Eszik I, Lantos I, Önder K, et al. High dynamic range detection of Chlamydia trachomatis growth by direct quantitative PCR of the infected cells. J Microbiol Methods. 2016;120:15-22. doi:10.1016/j. mimet.2015.11.010

29. Mossman T. Rapid colorimetric assay for cellular growth and survival: application to proliferation and cytotoxicity assays. J Immunol Meth. 1983;65(1-2):55-63.

30. Bhattacharjee S. DLS and zeta potential - what they are and what they are not? J Control Release. 2016;235:337-351. doi:10.1016/j. jconrel.2016.05.024

31. Chen Y, Lu Y, Chen J, et al. Enhanced bioavailability of the poorly watersoluble drug fenofibrate by using liposomes containing a bile salt. Int $J$ Pharm. 2009;376(1-2):153-160. doi:10.1016/j.ijpharm.2009.04.022

32. Hoppel M, Juric S, Ettl H, Valenta C. Effect of monoacyl phosphatidylcholine content on the formation of microemulsions and the dermal delivery of flufenamic acid. Int $J$ Pharm. 2015;479(1):7076. doi:10.1016/j.ijpharm.2014.12.048

33. Eggert-Kruse W, Kohler A, Rohr G, Runnebaum B. The $\mathrm{pH}$ as an important determinant of sperm-mucus interaction. Fertil Steril. 1993;59(3):617-628.

34. Martin C, Low WL, Gupta A, et al. Strategies for antimicrobial drug delivery to biofilm. Curr Pharm Des. 2015;21(1):43-66. doi:10.2174/ 1381612820666140905123529

35. Lai SK, Wang YY, Hanes J. Mucus-penetrating nanoparticles for drug and gene delivery to mucosal tissues. Adv Drug Deliv Rev. 2009;61(2):158-171. doi:10.1016/j.addr.2008.11.002

36. Van Bambeke F, Montenez JP, Piret J, Tulkens PM, Courtoy PJ, Mingeot-Leclercq MP. Interaction of the macrolide azithromycin with phospholipids. I. Inhibition of lysosomal phospholipase A1 activity. Eur J Pharmacol. 1996;314(1-2):203-214. doi:10.1016/ s0014-2999(96)00552-3

37. Jacobsen AC, Elvang PA, Bauer-Brandl A, Brandl M. A dynamic in vitro permeation study on solid mono- and diacyl-phospholipid dispersions of celecoxib. Eur J Pharm Sci. 2019;127:199-207. doi:10.1016/j.ejps.2018.11.003

38. Pavelić Ž, Škalko-Basnet N, Filipović-Grčić J, Martinac A, Jalšenjak I. Development and in vitro evaluation of a liposomal vaginal delivery system for acyclovir. J Control Release. 2005;106(1-2):34-43. doi:10.1016/j.jconrel.2005.03.032

39. Cadena PG, Pereira MA, Cordeiro RBS, et al. Nanoencapsulation of quercetin and resveratrol into elastic liposomes. Biochim Biophys Acta. 2013;1828(2):309-316. doi:10.1016/j.bbamem.2012.10.022

40. Machado D, Castro J, Palmeira-de-Oliveira A, Martinez-de-Oliveira J, Cerca N. Bacterial vaginosis biofilms: challenges to current therapies and emerging solutions. Front Microbiol. 2016;6:1528. doi:10.3389/fmicb.2015.01508

41. Jøraholmen MW, Vanić Ž, Tho I, Škalko-Basnet N. Chitosan-coated liposomes for topical vaginal therapy: assuring localized drug effect. Int J Pharm. 2014;472(1-2):94-101. doi:10.1016/j.ijpharm.2014. 06.016
42. Jøraholmen MW, Basnet P, Acharya G, Škalko-Basnet N. PEGylated liposomes for topical vaginal therapy improve delivery of interferon alpha. Eur J Pharm Biopharm. 2017;113:132-139. doi:10.1016/j. ejpb.2016.12.029

43. New RRC. Liposomes: A Practical Approach. Oxford: IRL Press; 1990.

44. Hoiby N, Bjarnsholt T, Givskov M, Molin S, Ciofu O. Antibiotic resistance of bacterial biofilms. Int J Antimicrob Agents. 2010;35 (4):322-332. doi:10.1016/j.ijantimicag.2009.12.011

45. Beaulac C, Sachetelli S, Lagace J. In-vitro bactericidal efficacy of sub-MIC concentrations of liposome-encapsulated antibiotic against gram-negative and gram-positive bacteria. $J$ Antimicrob Chemother. 1998;41(1):35-41. doi:10.1093/jac/41.1.35

46. Nicolosi D, Scalia M, Nicolosi VM, Pignatello R. Encapsulation in fusogenic liposomes broadens the spectrum of action of vancomycin against Gram-negative bacteria. Int J Antimicrob Agents. 2010;35 (6):553-558. doi:10.1016/j.ijantimicag.2010.01.015

47. Sun X, Qiu H, Jin Y. Highly efficient treatment of aerobic vaginitis with simple acidic buffered gels: the importance of $\mathrm{pH}$ and buffers on the micoenvironment of vaginas. Int J Pharm. 2017;525(1):175-182. doi:10.1016/j.jpharm.2017.04.026

48. Jones BM, Kinghorn GR, Duerden BI. In vitro activity of azithromycin and erythromycin against organisms associated with bacterial vaginosis and chancroid. Eur J Clin Microbiol Infect Dis. 1998;7 (4):551-553. doi:10.1007/BF01962614

49. De Backer E, Verhelst R, Verstraelen H, et al. Antibiotic susceptibility of Atopobium vaginae. BMC Infect Dis. 2006;6:51. doi:10.1186/1471-2334-6-51

50. Bretelle F, Fenollar F, Baumstarck K, et al. Screen-and-treat program by point-of-care of Atopobium vaginae and Gardnerella vaginalis in preventing preterm birth (AuTop trial): study protocol for a randomized controlled trial. Trials. 2015;16:470. doi:10.1186/s13063-015-0731-0

51. Wildfeuer A, Laufen H, Zimmermann T. Uptake of azithromycin by various cells and its intracellular activity under in vivo conditions. Antimicrob Agents Chemother. 1996;40(1):75-79. doi:10.1128/ AAC. 40.1 .75

52. Bosnar M, Kelnerić Z, Munić V, Eraković V, Parnham MJ. Cellular uptake and efflux of azithromycin, erythromycin, clarithromycin, telithromycin, and cethromycin. Antimicrob Agents Chemother. 2005;49(6):2372-2377. doi:10.1128/AAC.49.6.2372-2377.2005

53. Sangare L, Morisset R, Ravaoarinoro M. In-vitro anti-chlamydial activities of free and liposomal tetracycline and doxycycline. $J$ Med Microbiol. 1999;48(7):689-693. doi:10.1099/00222615-48-7-689

54. Zhou X, Zhang Y, Li Y, Hao X, Liu X, Wang Y. Azithromycin synergistically enhances anti-proliferative activity of vincristine in cervical and gastric cancer cells. Cancers. 2012;4(4):1318-1332. doi: $10.3390 /$ cancers 4041318

55. ISO10993-5, 2009. Biological evaluation of medical devices-part 5: tests for cytotoxicity: in vitro methods. Available from: https://www. iso.org/standard/36406.html. Accessed January 25, 2019.

56. Chang MC, Lee JJ, Chen YJ, et al. Lysophosphatidylcholine induces cytotoxicity/apoptosis and IL-8 production of human endothelial cells: related mechanisms. Oncotarget. 2017;8(63):106177-106189. doi:10.18632/oncotarget.22425

57. Alhajlan M, Alhariri M, Omri A. Efficacy and safety of liposomal clarithromycin and its effect on Pseudomonas aeruginosa virulence factors. Antimicrob Agents Chemother. 2013;57(6):2694-2704. doi:10.1128/AAC.00235-13 


\section{Publish your work in this journal}

The International Journal of Nanomedicine is an international, peerreviewed journal focusing on the application of nanotechnology in diagnostics, therapeutics, and drug delivery systems throughout the biomedical field. This journal is indexed on PubMed Central, MedLine, CAS, SciSearch ${ }^{\mathbb{R}}$, Current Contents ${ }^{(\mathbb{R}} /$ Clinical Medicine, $^{2}$
Journal Citation Reports/Science Edition, EMBase, Scopus and the Elsevier Bibliographic databases. The manuscript management system is completely online and includes a very quick and fair peer-review system, which is all easy to use. Visit http://www.dovepress.com/ testimonials.php to read real quotes from published authors.

Submit your manuscript here: https://www.dovepress.com/international-journal-of-nanomedicine-journal 